\title{
The topology of Stein fillable manifolds in high dimensions, II
}

\author{
JONATHAN BOWDEN \\ DiARMUID CROWLEY \\ ANDRÁS I STIPSICZ \\ APPENDIX BY BERND C KELLNER
}

\begin{abstract}
We continue our study of contact structures on manifolds of dimension at least five using surgery-theoretic methods. Particular applications include the existence of "maximal" almost contact manifolds with respect to the Stein cobordism relation as well as the existence of weakly fillable contact structures on the product $M \times S^{2}$. We also study the connection between Stein fillability and connected sums: we give examples of almost contact manifolds for which the connected sum is Stein fillable, while the components are not.
\end{abstract}

Concerning obstructions to Stein fillability, we show for all $k>1$ that there are almost contact structures on the $(8 k-1)$-sphere which are not Stein fillable. This implies the same result for all highly connected $(8 k-1)$-manifolds which admit almost contact structures. The proofs rely on a new number-theoretic result about Bernoulli numbers.

32E10; 57R17, 57R65

\section{Introduction}

One of the driving questions in contact topology was to determine which smooth closed oriented manifolds $M$ of dimension $2 q+1$ admit a contact structure, where a (coorientable) contact structure is a codimension-1 distribution $\xi$ that is defined as the kernel of a 1 -form $\lambda \in \Omega^{1}(M)$ with the property that $\lambda \wedge(d \lambda)^{q}$ is a positive volume form. Since a contact structure splits the tangent bundle of the $(2 q+1)$-manifold $M$ as the direct sum of a trivial real line bundle and a complex $q$-dimensional subbundle, we need to assume that the manifold in question is already equipped with such a splitting, called an almost contact structure. The general existence question for almost contact manifolds was recently answered by Borman, Eliashberg and Murphy: 
Theorem 1.1 [2] Suppose that $(M, \varphi)$ is a closed oriented $(2 q+1)$-dimensional almost contact manifold. Then there is a contact structure on $M$ homotopic to the given almost contact structure.

Indeed, the construction of [2] provides a contact structure which contains an overtwisted disk (cf [2, Section 2.5]), so in particular it is not symplectically fillable in any sense. (For various notions of symplectic fillability, see Section 2.1 and Massot, Niederkrüger and Wendl [24].) For this reason, constructions of fillable structures, and obstructions for their existence seem essential in an effort to understand all contact structures (up to contactomorphism or contact isotopy) on a given almost contact $(2 q+1)$-manifold. Such complete classifications are available for some classes of 3-dimensional manifolds, although the complete picture is still to be discovered even in that dimension.

The strongest fillability notion is provided by Stein fillability. Recall that a compact complex manifold $W$ is a Stein domain if it admits a strictly plurisubharmonic function for which the boundary is a regular level set. According to Eliashberg's characterization, a $2 n$-manifold with $n \geq 3$ admits a Stein structure if and only if it admits an almost complex structure and a handle decomposition with handles of index at most $n$ (see Cieliebak and Eliashberg [9] and Eliashberg [11]). A Stein structure on $W$ naturally induces a contact structure on $M=\partial W$, and contact structures presentable in this way are called Stein fillable. Using the above topological characterization of Stein domains, modified surgery theory can be fruitfully applied in studying Stein fillability as in Bowden, Crowley and Stipsicz [7].

This topological characterization of Stein domains easily generalizes to cobordisms, providing the relation of topological Stein cobordism for almost contact manifolds: Two almost contact manifolds $\left(M_{0}, \varphi_{0}\right)$ and $\left(M_{1}, \varphi_{1}\right)$ are in this relation if there is an almost complex cobordism between them that is compatible with $\varphi_{0}$ and $\varphi_{1}$ on the two ends, and admits a relative handle decomposition with handles of index at most half the dimension when built on $M_{0} \times[0,1]$. For convenience we write $\left(M_{0}, \varphi_{0}\right) \prec\left(M_{1}, \varphi_{1}\right)$ in this case. (Notice that this relation is not symmetric.)

A surprising application of the surgery-theoretic approach to existence problems in contact topology provides the following result about topological Stein cobordisms. (For a more precise statement, see Proposition 3.1.)

Theorem 1.2 For a fixed dimension $2 q+1 \geq 5$ there is an almost contact $(2 q+1)-$ manifold $\left(M_{\max }, \varphi_{\max }\right)$ such that for any almost contact $(2 q+1)$-manifold $(M, \varphi)$ we have

$$
(M, \varphi) \prec\left(M_{\max }, \varphi_{\max }\right) .
$$


This theorem should be compared with a result of Etnyre and Honda [12], showing that in dimension three there are initial contact manifolds so that $\left(M_{\min }, \xi_{\min }\right)$ is Stein cobordant to any other contact manifold $(M, \xi)$. In the case of almost contact 5manifolds whose almost contact structures have vanishing first Chern class, one can even take $\left(M_{\max }, \varphi_{\max }\right)=\left(S^{5}, \varphi_{\text {std }}\right)($ cf Proposition 3.4).

The notion of topological Stein cobordism introduced above allows for the following interpretation of the main result of Bowden, Crowley and Stipsicz [6]. Recall that according to a result of Bourgeois [4], for a contact manifold $(M, \xi)$ the product $M \times T^{2}$ carries an almost contact structure $\varphi_{T}$ which can be represented by a contact structure $\xi_{T}$. By Massot, Niederkrüger and Wendl [24, Example 5], if $(M, \xi)$ is weakly fillable then so is $\xi_{T}$. Since the main result of [6] shows that for some appropriately chosen almost contact structure $\varphi_{S}$ on $M \times S^{2}$ we have $\left(M \times T^{2}, \varphi_{T}\right) \prec\left(M \times S^{2}, \varphi_{S}\right)$, this yields the following variant of the main result of [6]:

Theorem 1.3 Suppose that a contact manifold $(M ; \xi)$ admits a weak symplectic filling $(W, \omega)$. Then the product $M \times S^{2}$ admits a weakly fillable contact structure.

Remark 1.4 Note that while all our other statements are concerned with almost contact structures on manifolds, Theorem 1.3 is about genuine contact structures.

We now move from products to connected sums. The connected sum of two 3-manifolds is Stein fillable if and only if both 3-manifolds are Stein fillable; see Eliashberg [10]. Recall that in higher dimensions, the diffeomorphism types of components of a connected sum are only well-defined up to connect summing with homotopy spheres. In contrast to dimension three, we have the following result.

Theorem 1.5 Let $M=S T^{*} S^{2 k+1}$ be the unit cotangent bundle of the $(2 k+1)-$ sphere. For every odd $k \geq 5, M$ admits an almost contact structure $\varphi$ such that $(M, \varphi) \#(-M,-\varphi)$ admits a Stein fillable contact structure. However, for every almost contact homotopy $(4 k+1)-$ sphere $\left(\Sigma, \varphi_{\Sigma}\right)$, neither $\left(M \# \Sigma, \varphi \# \varphi_{\Sigma}\right)$ nor $\left(-(M \# \Sigma),-\left(\varphi \# \varphi_{\Sigma}\right)\right)$ is Stein fillable.

Dimension five appears to be intermediate between dimension three and higher dimensions, with regard to the Stein fillability of the summands of a Stein fillable connected sum. In dimension five, there are no exotic spheres, and if $(M, \varphi)$ is an almost contact manifold where $M$ is a connected sum $M=M_{0} \# M_{1}$, then $(M, \varphi)=\left(M_{0}, \varphi\right) \#\left(M_{1}, \varphi_{1}\right)$ for almost contact structures $\varphi_{i}$ on $M_{i}$ which are uniquely defined up to homotopy (see Lemma 4.7). By abuse of notation we let

$$
c_{1}(\varphi): \pi_{2}(M) \rightarrow \mathbb{Z}=\pi_{2}(B U)
$$


denote the evaluation homomorphism given by the first Chern class of the almost contact structure $\varphi$. We then have the following analogue of Eliashberg's theorem.

Theorem 1.6 Let $(M, \varphi)=\left(M_{0} \# M_{1}, \varphi_{0} \# \varphi_{1}\right)$ be a Stein fillable almost contact 5-manifold. Assume that either $c_{1}(\varphi)=0$ or that

$$
c_{1}(\varphi)\left(\pi_{2}\left(M_{0}\right)\right)=c_{1}(\varphi)\left(\pi_{2}\left(M_{1}\right)\right)=\mathbb{Z}=\pi_{2}(B U) .
$$

Then both $\left(M_{0}, \varphi_{0}\right)$ and $\left(M_{1}, \varphi_{1}\right)$ are Stein fillable.

We next consider Stein fillability of almost contact structures on spheres. Denote by $\left(S^{2 q+1}, \zeta_{\text {std }}\right)$ the standard stable almost contact structure on the $(2 q+1)$-dimensional sphere, which is induced by the Stein $(2 q+2)-$ disk. When $2 q+1=8 k-1$, basic obstruction theory shows that $S^{8 k-1}$ has two stable almost contact structures, $\zeta_{\text {std }}$ and a nonstandard or exotic stable almost contact structure $\zeta_{\text {ex }}$. The exotic structure $\zeta_{\mathrm{ex}}$ is harder to visualize than $\zeta_{\text {std }}$ (see Section 5.2 for a description when $k>1$ ). It follows from Bowden, Crowley and Stipsicz [7] and Geiges [13] that $\left(S^{7}, \zeta_{\text {ex }}\right)$ can be represented by a Stein fillable contact structure. In contrast, for $8 k-1>7$ we have the following theorem.

Theorem 1.7 The exotic stable almost contact structure $\zeta_{\mathrm{ex}}$ on $S^{8 k-1}$ cannot be represented by a Stein fillable contact structure once $k \geq 2$.

Theorem 1.7 rests on Theorem 1.8 below, which improves a result of Yang [34] about the existence of stable almost complex structures on $(4 k-1)-$ connected $8 k-$ manifolds. Before stating these results we first recall some notation and terminology. Let $F: B U \rightarrow B$ SO be the forgetful map between the classifying spaces for stable unitary and stable oriented vector bundles. A necessary condition for an oriented manifold $X$ to admit a stable complex structure is that

$$
\operatorname{Im}\left(\tau_{X *}\right) \subseteq F_{*}\left(\pi_{4 k}(B U)\right) \subseteq \pi_{4 k}(B \mathrm{SO}),
$$

where $\tau_{X *}: \pi_{4 k}(X) \rightarrow \pi_{4 k}(B \mathrm{SO})$ is induced by the classifying map of the stable tangent bundle of $X, \tau_{X}: X \rightarrow B$ SO. (Note that $\pi_{4 k}(B S O) / F_{*}\left(\pi_{4 k}(B U)\right)=\mathbb{Z} / 2$ when $k$ is even.) According to the following theorem, once $k>1$, this necessary condition is also sufficient.

Theorem 1.8 A smooth closed oriented (4k-1)-connected $8 k$-manifold $Y$ admits a stable almost complex structure if and only if:

(1) $k \geq 3$ is odd.

(2) $k=1$ and the signature $\sigma_{Y}$ of $Y$ is even.

(3) $k$ is even and $\operatorname{Im}\left(\tau_{Y *}\right) \subseteq F_{*}\left(\pi_{4 k}(B U)\right)$. 
The improvement provided by Theorem 1.8 over Yang's result is the removal of assumptions involving Bernoulli numbers. This step is made possible by a new divisibility property of differences of reciprocals of Bernoulli numbers, which is proven in the appendix written by Bernd Kellner.

Computing the appropriate bordism obstruction class to Stein fillability from [7], Theorem 1.7 implies the following nonfillability result for highly connected manifolds:

Corollary 1.9 Let $M$ be a (4k-2)-connected ( $8 k-1)$-manifold and $k \geq 2$. Suppose that $M$ admits an almost contact structure. Then $M$ admits an almost contact structure which cannot be represented by any Stein fillable contact structure.

Outline of the paper In Section 2 we review some basic notions and recall the definition of the obstruction class as introduced in [7] associated to an almost contact manifold. We also prove Theorem 1.3 in this section. In Section 3 we present the proof of Theorem 1.2. Section 4 is devoted to the study of the relation between Stein fillability and connected sums, and in particular it contains the proofs of Theorems 1.5 and 1.6. In Section 5 we examine the Stein fillability of stable almost contact structures on $(8 k-1)-$ spheres, and in particular prove Theorem 1.7 and Corollary 1.9. Section 5 also contains our improvement of Yang's result given in Theorem 1.8 about the existence of stable almost complex structures on highly connected $8 k$-manifolds. The appendix contains the number-theoretic result about Bernoulli numbers needed for the proof of Theorem 1.7, and was written by Bernd Kellner.

Acknowledgements The authors would like to thank the Max-Planck-Institute in Bonn and the Laboratoire de Mathématiques Jean Leray in Nantes for their hospitality which enabled parts of this work to be carried out. We would also like to thank Pieter Moree for providing a bridge to the world of number theory and contacting Karl Dilcher and Bernd Kellner. We are grateful to the referee for many helpful comments and suggestions. JB was partially supported by DFG Grant BO4423/1-1. DC acknowledges the support of the Leibniz Prize of Wolfgang Lück, granted by the Deutsche Forschungsgemeinschaft. AS was partially supported by OTKA K100796, by the Lendület program of the Hungarian Academy of Sciences and by ERC Advanced Grant LDTBud. The present work is part of the authors' activities within CAST, a Research Network Program of the European Science Foundation.

\section{Fillability and surgery}

In their proof of the existence of contact structures on closed almost contact manifolds, Borman, Eliashberg and Murphy [2] produce contact structures with the additional 
property that they are not fillable in any sense. For this reason we will focus on finding fillable structures on various manifolds.

\subsection{Fillable structures and Stein cobordisms}

We begin by recalling the definitions of the various standard notions of fillability of contact structures. For a more detailed account we refer to $[15 ; 24]$. Recall that a symplectic manifold $(W, \omega)$ is a $(2 q+2)$-dimensional manifold $W$ with a closed 2 -form $\omega$ such that $\omega^{q+1} \neq 0$. Hence a symplectic manifold carries a canonical orientation. Similarly, a cooriented contact structure $\xi$ on a $(2 q+1)-$ manifold $M$ determines an orientation of $M$ given by the form $\lambda \wedge(d \lambda)^{q}$.

Definition 2.1 A contact manifold $(M, \xi)$ is weakly symplectically fillable if it is the oriented boundary of a compact symplectic manifold $(W, \omega)$ and there is an almost complex structure $J$ that is tamed by $\omega$ so that $J(T M) \cap T M=\xi$ and for a contact form $\lambda$ defining $\xi$ we have $d \lambda(v, J v)>0$ (for all $0 \neq v \in \xi$ ).

This definition was introduced in [24], where it was shown to be strictly weaker than the more standard notion of strong fillability.

Definition 2.2 A contact manifold $(M, \xi)$ is called strongly symplectically fillable if it bounds a compact symplectic manifold $(W, \omega)$ and there is an outward pointing vector field $V$ near $\partial W$ such that the Lie derivative satisfies $L_{V} \omega=\omega$ and $\lambda=\iota_{V} \omega$ is a defining 1 -form for $\xi$. If the symplectic form $\omega$ is also exact then we say that $(M, \xi)$ is exactly fillable.

Note that strong fillability is equivalent to weak fillability plus the condition that the symplectic form is exact near the boundary [24, Remark 1.11]. A further specialization of the fillability notion is that of Stein fillability. Recall that a Stein domain is a compact, complex manifold $(W, J)$ with boundary that admits a function $f: W \rightarrow[0,1]$ so that $f^{-1}(1)=\partial W$ is a regular level set and $\omega=-d d^{\mathbb{C}} f$ is a symplectic form (where $\left.d^{\mathbb{C}} f(X)=d f(J X)\right)$.

Definition 2.3 A contact manifold $(M, \xi)$ is Stein fillable if it bounds a Stein domain $(W, J)$ such that $\xi=J(T M) \cap T M$.

These notions of fillability fit into the following sequence of inclusions of contactomorphism classes of contact manifolds, all of which are known to be strict:

$\{$ Stein fillable $\} \subset\{$ exactly fillable $\} \subset\{$ strongly fillable $\} \subset\{$ weakly fillable $\}$.

The applicability of surgery-theoretic methods in the study of fillable contact structures is provided by the following fundamental result of Eliashberg: 
Theorem 2.4 (Eliashberg's $h$-principle $[9 ; 11])$ Let $(W, J)$ be a compact $(2 q+2)$ dimensional almost complex manifold admitting a handle decomposition with handles of index $q+1$ or less, and suppose that $q \geq 2$. Then $J$ is homotopic to a complex structure $\widetilde{J}$ so that $(W, \widetilde{J})$ is a Stein filling of a contact structure $\xi$ on $M=\partial W$.

The concept of Stein domains can be generalized to cobordisms as follows:

Definition 2.5 A smooth cobordism $W$ between contact manifolds $\left(M_{0}, \xi_{0}\right)$ and $\left(M_{1}, \xi_{1}\right)$ is a Stein cobordism if:

- $\partial W=-M_{0} \sqcup M_{1}$.

- $W$ admits a complex structure $J$ and a map $f: W \rightarrow[0,1]$ such that $M_{0}:=$ $f^{-1}(0)$ and $M_{1}:=f^{-1}(1)$ are regular level sets.

- $\omega=-d d^{\mathbb{C}} f$ is a symplectic form.

- $\xi_{i}=J\left(T M_{i}\right) \cap T M_{i}$, ie the complex structure $J$ induces the contact structures $\xi_{i}$ on the ends of the cobordism. The contact manifold $\left(M_{0}, \xi_{0}\right)$ is usually called the concave end and $\left(M_{1}, \xi_{1}\right)$ the convex end of the Stein cobordism $(W, J)$.

The proof of Theorem 2.4 proceeds by inductively adding handles to the standard contact structure on the sphere $S^{2 q+1}$ (which is regarded as the boundary of the standard complex ball), and showing that the traces of these handle attachments can be endowed with the structure of a Stein cobordism:

Theorem 2.6 Let $\left(M^{2 q+1}, \xi\right)$ be a contact manifold of dimension $2 q+1 \geq 5$. Suppose that $k \leq q+1$ and that $M^{\prime}$ is obtained from $M$ via an almost complex handle attachment of index $k$ with trace $(M \times I) \cup h_{k}$. Then the almost complex structure $J$ on the trace is homotopic to a complex structure $\widetilde{J}$ so that $\left((M \times I) \cup h_{k}, \widetilde{J}\right)$ is a Stein cobordism from $(M, \xi)$ to $\left(M^{\prime}, \xi^{\prime}\right)$ (with some contact structure $\xi^{\prime}$ on $M^{\prime}$ ).

Remark 2.7 By equipping the product $M_{0} \times I$ with the symplectic structure given by the symplectization $\omega_{s p}\left(\xi_{0}\right)$ of the contact structure $\xi_{0}$ and isotoping the attaching sphere of the handle $h_{k}$ to an isotropic sphere, the symplectic form $\omega_{s p}\left(\xi_{0}\right)$ was extended by Weinstein [32] to the trace $\left(M_{0} \times I\right) \cup h_{k}$. The existence of a Stein structure on the trace (in particular, the construction of the appropriate function $f$ of the definition) is due to Eliashberg $[9 ; 11]$. When the symplectic or Stein structures are implicitly assumed in our later arguments, we will refer to such handles and handle attachments as Stein/Weinstein handles resp. handle attachments. 
In the following we would like to emphasize the topological nature of the above definitions. To do this in the proper setting, we need to recall the definitions of almost contact and stably complex structures and manifolds.

Suppose that $M$ is a smooth closed oriented $(2 q+1)$-manifold and $\varphi$ is an almost contact structure on $M$. The tangent bundle of $M$ is classified by the map $\tau: M \rightarrow$ $B \mathrm{SO}(2 q+1)$, and an almost contact structure provides a lift of this map to $B U(q)$ :

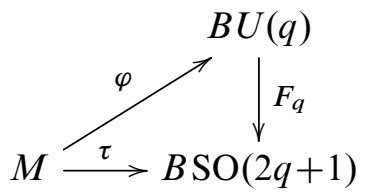

Here $F_{q}$ is induced by the canonical embedding $U(q) \rightarrow \mathrm{SO}(2 q+1)$. All these maps can be stabilized to yield maps to $B \mathrm{SO}$ resp. $B U$. For some purposes, it is helpful to formulate results using the stable normal Gauss map $v: M \rightarrow B$ SO rather than the tangential map $\tau$, and we will follow this strategy. In this setting, a map $\zeta: M \rightarrow B U$ in a commutative diagram

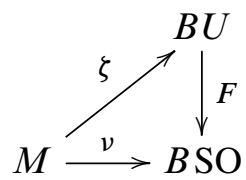

describes a complex structure on the normal bundle of $M$. Since the sum of the stable tangent and normal bundles is canonically trivialized, a normal complex structure determines a unique stable complex (or stable contact) structure, and vice versa. Theorem 2.6 motivates the following definition:

Definition 2.8 A stably almost contact $(2 q+1)$-manifold $\left(M_{0}, \zeta_{0}\right)$ is topologically Stein cobordant to $\left(M_{1}, \zeta_{1}\right)$ if there is a stably complex cobordism $(W, \zeta)$ such that

$$
\partial(W, \zeta)=-\left(M_{0}, \zeta_{0}\right) \sqcup\left(M_{1}, \zeta_{1}\right)
$$

as stably complex manifolds and $W$ is built from $M_{0} \times[0,1]$ by attaching handles of index $\leq q+1$. In this case we write

$$
\left(M_{0}, \zeta_{0}\right) \prec\left(M_{1}, \zeta_{1}\right)
$$

and call $(W, \zeta)$ a topological Stein cobordism.

Note that according to [7, Lemma 3.6] the Stein cobordism relation is the same if we consider almost complex cobordisms or stably complex ones. This follows from the fact that every almost complex structure in a given stable class can be realized by taking 
the connected sum with various Stein fillable almost contact structures on the standard sphere. Hence we can also consider the Stein cobordism relation given by a true almost complex bordism $(W, J)$ between almost contact manifolds $\left(M_{0}, \varphi_{0}\right)$ and $\left(M_{1}, \varphi_{1}\right)$. In short, if $\varphi_{i}$ generates the stable complex structure $\zeta_{i}$, then

$$
\left(M_{0}, \varphi_{0}\right) \prec\left(M_{1}, \varphi_{1}\right) \Longleftrightarrow \quad\left(M_{0}, \zeta_{0}\right) \prec\left(M_{1}, \zeta_{1}\right)
$$

If, in addition, an almost contact structure $\varphi_{0}$ on $M_{0}$ is represented by a contact structure, then repeated application of Theorem 2.6 shows that $J$ can be homotoped to a Stein structure on the cobordism $W$.

A Stein cobordism from $\left(M_{0}, \xi_{0}\right)$ to $\left(M_{1}, \xi_{1}\right)$ can be glued to a Stein filling of $\left(M_{0}, \xi_{0}\right)$, providing a Stein filling of $\left(M_{1}, \xi_{1}\right)$. Attaching Stein/Weinstein handles preserves strong fillability, hence gluing a Stein cobordism to a strong filling again yields a strong filling. When gluing a Stein cobordism to a weak symplectic filling, however, some care is needed: as shown by the next lemma, we need to assume that the symplectic form vanishes on the attaching spheres of 3-handles.

Lemma 2.9 Let $(W, \omega)$ be a weak filling of a contact manifold $\left(M, \xi_{0}\right)$ and suppose that $\left(W_{1}, J\right)$ is a Stein cobordism from $\left(M, \xi_{0}\right)$ to $\left(M_{1}, \xi_{1}\right)$ consisting of a single $k$-handle attachment so that $\omega$ vanishes on the homology class of the attaching sphere if $k=3$. Then $W^{\prime}=W \cup W_{1}$ (equipped with a suitable symplectic structure $\omega^{\prime}$, based on $\omega$ and the Stein structure on $\left.W_{1}\right)$ provides a weak filling of $\left(M_{1}, \xi_{1}\right)$.

Furthermore, if the attaching sphere of a 2-handle bounds a surface $\Sigma$ in $M$ then we can assume that the $\omega^{\prime}\left(\left[\Sigma \cup D^{2}\right]\right)=0$, where $D^{2}$ denotes the core of the 2-handle.

Proof Let $(-\epsilon, \epsilon) \times M$ be a small regular neighbourhood of $M$ in $W$, where $W$ has been extended slightly. Let $\lambda$ be a defining 1-form for $\xi$. Suppose that $\left.\omega\right|_{M}$ is exact on the attaching sphere $S^{k-1}$ of the $k$-handle of $W_{1}$. Then there is a form $\bar{\omega}$ cohomologous to $\left.\omega\right|_{M}$ which vanishes near $S^{k-1}$. By [24, Lemma 1.10] one can alter the symplectic structure after attaching a sufficiently long end $[0,2 C] \times \partial W$ so that the symplectic form is given by $\bar{\omega}+d(t \lambda)$ for all $t \geq C-\epsilon$ and we still have a weak filling of $M=M \times\{C\}$. In particular, near $S^{k-1} \subset M \times\{C\}$ the symplectic form is just $d(t \lambda)$. We attach a Stein/Weinstein $k$-handle along $S^{k-1}$ and denote the resulting filling by $\left(W^{\prime}, \omega^{\prime}\right)$. The almost complex structure $J$ on $W$ used in the definition of weak filling then extends to an almost complex structure $J^{\prime}$ on $W^{\prime}$ that is tamed by $\omega^{\prime}$.

Since $\omega$ is always exact near an attaching sphere $S^{k-1}$ with $k \neq 3$ and this is the case by assumption if $k=3$, the lemma follows immediately. 
In the case of a 2 -handle whose attaching sphere $S^{1}$ bounds a surface $\Sigma$, we can assume that the form $\bar{\omega}$ above vanishes on $\Sigma \subset M \times\{C\}$. Then since the core of a Weinstein handle is isotropic with isotropic boundary, it follows that

$$
\omega^{\prime}\left(\left[\Sigma \cup D^{2}\right]\right)=\int_{\Sigma} C d \lambda+\int_{D^{2}} \omega^{\prime}=\int_{\partial \Sigma} C \lambda=0,
$$

giving the final claim.

In [6], a contact structure was constructed on $M \times S^{2}$ by constructing a topological Stein cobordism between $M \times T^{2}$ and $M \times S^{2}$ for appropriate choices of almost contact structures. With the above lemma at hand, this point of view then provides the following fillability result, which corresponds to Theorem 1.3 in Section 1.

Proposition 2.10 Let $(M, \xi)$ be a contact manifold of dimension $2 q+1$ that admits a weak symplectic filling $(W, \omega)$. Then $M \times S^{2}$ admits a weakly fillable contact structure.

Proof Let $(W, \omega)$ be a weak filling of $(M, \xi)$. By [24, Example 5] the manifold $M \times T^{2}$ admits a contact structure that is weakly filled by the symplectic manifold $\left(W \times T^{2}, \omega \oplus \omega_{T^{2}}\right)$. According to [6, Proposition 3.1] there is a Stein cobordism $Y$ from $M \times T^{2}$ to $M \times S^{2}$ which fits into the following diagram:



where $g_{Y}$ is a $(q+2)$-equivalence and $f_{0}$ is the product of the identity with a map of degree 1 .

The idea of the proof is to inductively apply Lemma 2.9 to Stein handle attachments which make up the bordism $Y$, starting from from $M \times T^{2}$. We first find a topological Stein structure on $Y$ where we can apply Lemma 2.9 to each handle attachement. For this, we need to keep track of the cohmology class of the symplectic form of the filling, when restricted to the outgoing boundary. Hence we note the equality of cohomology classes $\left[\left.\omega\right|_{M} \oplus \omega_{T^{2}}\right]=f_{0}^{*}\left(\left[\left.\omega\right|_{M} \oplus \omega_{S^{2}}\right]\right)$ for a symplectic form $\omega_{S^{2}}$ on $S^{2}$.

Let $\alpha, \beta \subset T^{2}$ be the standard generators of $\pi_{1}\left(T^{2}\right)$ which we consider in different $T^{2}$-fibers of $M \times T^{2}$. These are then null-homotopic in $Y$ and hence extend to maps of discs, which can be taken to be proper embeddings in $Y$ since the dimension of $Y$ is at least 6 . Let $Y_{2}$ be the bordism obtained by attaching a pair of 2 -handles along $\alpha$ and 
$\beta$. We then obtain decomposition of $Y=Y_{2} \cup_{X_{2}} Y_{3}$, where $X_{2}$ is the upper boundary component of $Y_{2}$. Attaching these 2-handles yields $\pi_{1}\left(Y_{2}\right)=\pi_{1}\left(X_{2}\right)=\pi_{1}(M)$ by construction and also that the map $Y_{2} \rightarrow M \times S^{2}$ is a surjection on $\pi_{2}$. This latter claim in obvious for classes in the $\pi_{2}(M)$-factor and for the class coming form the $S^{2}$-factor observe that the result of the surgery on the class $\left[\mathrm{pt} \times T^{2}\right]$ is a spherical class that is mapped to $\left[\mathrm{pt} \times S^{2}\right]$ under $\left(g_{Y}\right)_{*}$.

Since $Y_{2}$ is formed by 2-handle attachments, it has the homotopy type of a space obtained by attaching $(2 q+2)-$ cells to $X_{2}$. Hence the inclusion $X_{2} \rightarrow Y_{2}$ is $(2 q+1)-$ connected and so $g_{X_{2}}: X_{2} \rightarrow M \times S^{2}$ is 2-connected: Here, and for the rest of the proof, we set $g_{Z}:=\left.g_{Y}\right|_{Z}$ for any subspace $Z \subset Y$. Since $Y_{3}$ is obtained from the $(2 q+2)$-dimensional manifold $Y$ by deleting neighbourhoods of 2-handles, the inclusion $Y_{3} \rightarrow Y$ is $(2 q+1)$-connected and so $g_{Y_{3}}: Y_{3} \rightarrow M \times S^{2}$ is at least 3connected. It follows that the pair $\left(Y_{3}, X_{2}\right)$ is algebraically 2 -connected, and we will use this later in the proof.

The fact that $Y_{3}$ is obtained from $Y$ by deleting neighbourhoods of 2-handles has another important consequence. Combined with the fact that $\left(Y, M \times S^{2}\right)$ is algebraically $(q+1)$-connected, it implies that the pair $\left(Y_{3}, M \times S^{2}\right)$ is algebraically $(q+1)$-connected. Thus there is a handle decomposition of $Y_{3}$ relative to $M \times S^{2}$ containing only handles of index at least $q+2$ by a result of Wall [7, Theorem 2.18] or dually there is a handle decomposition of $Y_{3}$ relative to $X_{2}$ containing only handles of index at most $q+2$. More precisely Wall shows inductively that given any handle decomposition one can cancel handles of index $k<q+2$ with $(k+1)$-handles at the expense of introducing a $(k+2)$-handles.

We now apply Lemma 2.9 to $Y_{2}$ to obtain a weak filling $\left(Y_{2}, \omega^{\prime}\right)$ of $X_{2}$. Since the 2-handles are attached along curves that are non-trivial in rational homology it follows from the long exact sequence of the pair $\left(Y_{2}, M \times T^{2}\right)$ that the map $M \times T^{2} \rightarrow Y_{2}$ induces an injection on cohomology. In particular, we have the following equality for cohomology classes $\left[\omega^{\prime}\right]=\left(g_{Y_{2}}\right)^{*}\left(\left[\left.\omega\right|_{M} \oplus \omega_{S^{2}}\right]\right)$.

We now consider any topological Stein handle decomposition of $Y_{3}$ relative to $X_{2}$. As this pair is 2-connected we can then apply Wall's argument to cancel all handles of index $k \leq 1$ at the expense of introducing 3-handles. In particular, the resulting handle decomposition will still be topologically Stein as $\operatorname{dim}\left(Y_{3}\right)=2 q+4 \geq 6$.

We now attach the 2-handles $h_{i}^{2}$ of $Y_{3}$ to $X_{2}$. Let $Y_{2+}=Y_{2} \cup \bigcup_{i=1}^{n} h_{i}^{2} \subset Y_{3}$ be the union of $Y_{2}$ and these new 2-handles. Let $D_{i}^{2}$ be the core of $h_{i}^{2}$. Since the pair $\left(Y_{3}, X_{2}\right)$ is 2-connected, the disc $D_{i}^{2}$ can be homotoped inside $Y_{3}$ to a disc $\Delta_{i} \subset X_{2}$ relative to its boundary, which by general position is embedded. Applying Lemma 2.9 for each 2-handle we obtain a weak filling $\left(Y_{2+}, \omega^{\prime}\right)$ such that $\omega^{\prime}\left(\left[D_{i}^{2} \cup \Delta_{i}\right]\right)=0$. We claim 
that at the level of cohomology classes we again have $\left[\omega^{\prime}\right]=\left(g_{Y_{2+}}\right)^{*}\left(\left[\left.\omega\right|_{M} \oplus \omega_{S^{2}}\right]\right)$. To see this, consider the cohomology exact sequence of the pair $\left(Y_{2+}, Y_{2}\right)$ :

$$
\cdots \longrightarrow H^{2}\left(Y_{2+}, Y_{2}\right) \stackrel{j}{\longrightarrow} H^{2}\left(Y_{2+}\right) \longrightarrow H^{2}\left(Y_{2}\right) \longrightarrow \cdots .
$$

By construction, $\left[\omega^{\prime}\right]$ and $\left(g_{Y_{2+}}\right)^{*}\left(\left[\left.\omega\right|_{M} \oplus \omega_{S^{2}}\right]\right)$ agree when restricted to $Y_{2}$, so their difference lies in $j\left(H^{2}\left(Y_{2+}, Y_{2}\right)\right)$, where $H^{2}\left(Y_{2+}, Y_{2}\right)$ is a free abelian group with dual basis consisting of the $2-$ handles $h_{i}^{2}$. Since $D_{i}^{2}$ and $\Delta_{i}$ are homotopic relative to their boundary in $Y_{3}$, the spherical class $\left[D_{i}^{2} \cup \Delta_{i}\right]$ is null-homotopic in $Y_{3}$. This, combined with the fact that $\omega^{\prime}\left(\left[D_{i}^{2} \cup \Delta_{i}\right]\right)=0$, ensures that

$$
\left[\omega^{\prime}\right]-\left(g_{Y_{2+}}\right)^{*}\left(\left[\left.\omega\right|_{M} \oplus \omega_{S^{2}}\right]\right)=0 .
$$

We next attach 3-handles to $Y_{2+}$. In order to apply Lemma 2.9 we must ensure that the resulting symplectic form vanishes on the attaching $2-$ sphere $S_{a}^{2}$. Let $\iota_{2+}: Y_{2+} \rightarrow Y$ be the inclusion. Since $S_{a}^{2}$ bounds a 3 -disc in $Y$, it follows that

$$
\left(g_{Y_{2+}}\right)_{*}\left(\left[S_{a}^{2}\right]\right)=\left(g_{Y}\right)_{*}\left(\iota_{2+}\right)_{*}\left(\left[S_{a}^{2}\right]\right)=0 \in H_{2}\left(M \times S^{2}\right) .
$$

We then have

$$
\left\langle\left(g_{Y_{2+}}\right)^{*}\left(\left[\left.\omega\right|_{M} \oplus \omega_{S^{2}}\right]\right),\left[S_{a}^{2}\right]\right\rangle=\left\langle\left[\left.\omega\right|_{M} \oplus \omega_{S^{2}}\right],\left(g_{Y_{2+}}\right)_{*}\left(\left[S_{a}^{2}\right]\right)\right\rangle=0,
$$

where the angular brackets denote the natural Kronecker pairing. Thus we can again apply Lemma 2.9. As above the cohomology class of the symplectic structure $\omega^{\prime}$ is just the restriction of $\left(g_{Y}\right)^{*}\left(\left[\left.\omega\right|_{M} \oplus \omega_{S^{2}}\right]\right)$. Inductively applying Lemma 2.9 to the remaining handles completes the argument.

\subsection{The surgery obstruction and topological Stein cobordisms}

In this subsection we briefly recall the main construction of [7]. We then extend this point of view and identify the "topological Stein envelope" of an almost contact manifold, ie those almost contact manifolds which can be obtained from a given one via a topological Stein cobordism.

Recall that an almost contact structure $\varphi$ on a $(2 q+1)$-manifold $M$ can be regarded as a map $\varphi: M^{2 q+1} \rightarrow B U(q)$, which lifts the classifying map $\tau: M^{2 q+1} \rightarrow B \mathrm{SO}(2 q+1)$ of the tangent bundle of $M$. We then stabilize $\varphi$ and pass to the corresponding complex normal structure, which is an equivalence class of maps $\zeta: M \rightarrow B U$, which lift the stable normal Gauss map $v: M \rightarrow B$ SO. 
For a fixed integer $k$, the map $\zeta: M \rightarrow B U$ admits a Postnikov factorization $\left(B_{\zeta}^{k}, \eta_{\zeta}^{k}, \bar{\zeta}\right)$ with the following properties: these maps and spaces fit into the commutative diagram

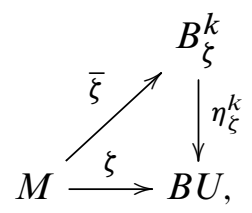

and satisfy the following conditions:

(1) $\eta_{\zeta}^{k}$ is a Serre fibration.

(2) $\bar{\zeta}$ is a $(k+1)$-equivalence, that is, it induces an isomorphism on $\pi_{i}$ for all $i<k+1$ and a surjection for $i=k+1$.

(3) $\eta_{\zeta}^{k}$ is a $(k+1)$-coequivalence, that is, it induces an isomorphism on $\pi_{i}$ for $i>k+1$ and an injection for $i=k+1$.

The existence of these spaces and maps are proved in [1, Chapters 2 and 5]. The pair $\left(B_{\zeta}^{k}, \eta_{\zeta}^{k}\right)$ is unique (up to fiber homotopy equivalence), and we call them the complex normal $k$-type of the stable complex manifold $(M, \zeta)$. The map $\bar{\zeta}: M \rightarrow B_{\zeta}^{k}$ is called a $\zeta$-compatible normal smoothing and is not, in general, uniquely determined by $\zeta$. The only explicit complex normal $k$-types we will use in this paper are covered by the following example.

Example 2.11 (cf [7, Example 2.5]) We take the stable complex bundle

$$
\left(B_{\zeta}^{k}, \eta_{\zeta}^{k}\right)=\left(B U\langle k+1\rangle, \pi_{k+1}\right)
$$

where the map $\pi_{k+1}: B U\langle k+1\rangle \rightarrow B U$ is the $k$-fold connective covering of $B U$. Recall that $B U\langle k+1\rangle$ is the space whose homotopy groups are trivial in degree $i \leq k-1$ and such that $\pi_{k+1}$ induces a surjection on the $k^{\text {th }}$ homotopy group and isomorphisms for all higher homotopy groups. We denote the bordism groups $\Omega_{*}\left(B U\langle k+1\rangle ; \pi_{k+1}\right)$ by $\Omega_{*}^{U\langle k\rangle}$. When $k=3$, we have that $\Omega_{*}\left(B U\langle 4\rangle ; \pi_{4}\right)=\Omega_{*}^{\text {SU }}$ is just special unitary bordism as considered in [30, Chapter $\mathrm{X}]$.

For an almost contact $(2 q+1)$-manifold $(M, \varphi)$ with its induced stable complex structure $\zeta$ we consider the associated complex normal $(q-1)$-type $\left(B_{\zeta}^{q-1}, \eta_{\zeta}^{q-1}\right)$. The map $\bar{\zeta}$ then provides a bordism class $[M, \bar{\zeta}]$ in the bordism group

$$
\Omega_{2 q+1}\left(B_{\zeta}^{q-1} ; \eta_{\zeta}^{q-1}\right)
$$

For a detailed discussion of this group, see [7]. 
A priori the bordism class $[M, \bar{\zeta}]$ depends on the choice of $(q-1)$-smoothing $\bar{\zeta}$; we call any such class an obstruction class, since - according to the next theorem $-[M, \bar{\zeta}]$ vanishes if and only if the almost contact structure $\varphi$ can be represented by a Stein fillable contact structure.

Theorem 2.12 [7, Theorem 1.2] A closed almost contact manifold $(M, \varphi)$ of dimension $2 q+1 \geq 5$ admits a Stein fillable contact structure homotopic to the almost contact structure $\varphi$ if and only if

$$
[M, \bar{\zeta}]=0 \in \Omega_{2 q+1}\left(B_{\zeta}^{q-1} ; \eta_{\zeta}^{q-1}\right)
$$

for any, equivalently for all, choices of $\bar{\zeta}$, where $\zeta$ is the stabilization of $\varphi$.

Remark 2.13 The applicability of the obstruction class described above hinges on computations of the bordism group

$$
\Omega_{2 q+1}\left(B_{\zeta_{1}}^{q-1} ; \eta_{\zeta_{1}}^{q-1}\right)
$$

which is a highly nontrivial matter in general. For simply connected 7-manifolds with torsion free second homotopy group [7, Theorem 1.3] shows that

$$
\Omega_{7}\left(B_{\zeta}^{2}, \eta_{\zeta}^{2}\right)=0
$$

implying that all such almost contact 7 -manifolds are Stein fillable. For $(q-1)-$ connected $(2 q+1)$-manifolds further calculations of these bordism groups will be presented in [5].

In terms of the topological Stein cobordism relation from Definition 2.8, Theorem 2.12 states that $\left(S^{2 k+1}, \zeta_{\text {std }}\right) \prec(M, \zeta)$ if and only if an obstruction class $[M, \bar{\zeta}]$ vanishes. We now extend Theorem 2.12 to give a bordism-theoretic determination of the topological Stein cobordism relation for any pair of closed $(2 q+1)$-dimensional stably complex manifolds $\left(M_{0}, \zeta\right)$ and $\left(M_{1}, \zeta_{1}\right)$.

Theorem 2.14 There is a topological Stein cobordism $(W, \zeta)$ from $\left(M_{0}, \zeta_{0}\right)$ to $\left(M_{1}, \zeta_{1}\right)$, ie $\left(M_{0}, \zeta_{0}\right) \prec\left(M_{1}, \zeta_{1}\right)$, if and only if there is a map $\alpha$ of fibrations over $B U$

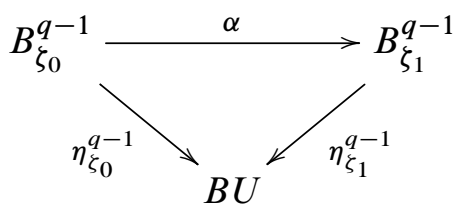

and $\zeta_{i}$-compatible normal $(q-1)$-smoothings $\bar{\zeta}_{i}: M_{i} \rightarrow B_{\zeta_{i}}^{q-1}$ such that

$$
\alpha_{*}\left(\left[M_{0}, \bar{\zeta}_{0}\right]\right)=\left[M_{1}, \bar{\zeta}_{1}\right] \in \Omega_{2 q+1}\left(B_{\zeta_{1}}^{q-1} ; \eta_{\zeta_{1}}^{q-1}\right)
$$


Proof Suppose that

$$
\alpha_{*}\left(\left[M_{0}, \bar{\zeta}_{0}\right]\right)=\left[M_{1}, \bar{\zeta}_{1}\right]
$$

and let $(W, \bar{\zeta})$ be a $\left(B_{\zeta_{1}}^{q-1}, \eta_{\zeta 1}^{q-1}\right)-$ nullcobordism of $\left(-M_{0},-\alpha \circ \bar{\zeta}_{0}\right) \sqcup\left(M_{1}, \bar{\zeta}_{1}\right)$. Applying surgery below the middle dimension [7, Proposition 2.6], we can assume that $\bar{\zeta}: W \rightarrow B_{\zeta_{1}}^{q-1}$ is a $(q+1)$-equivalence. It follows from a result of Wall [7, Theorem 2.18] that $W$ is built from $M_{1}$ by attaching handles of index $\geq q+1$ and dually that $W$ is obtained from $M_{0}$ by attaching handles of index at most $q+1$, verifying one direction of the equivalence.

Conversely, if $(W, \zeta)$ is a topological Stein cobordism with boundary $\left(-M_{0},-\zeta_{0}\right) \sqcup$ $\left(M_{1}, \zeta_{1}\right)$, then the universal properties of Postnikov factorizations [1, Chapters 2 and 5] mean that there is a homotopy commutative diagram,

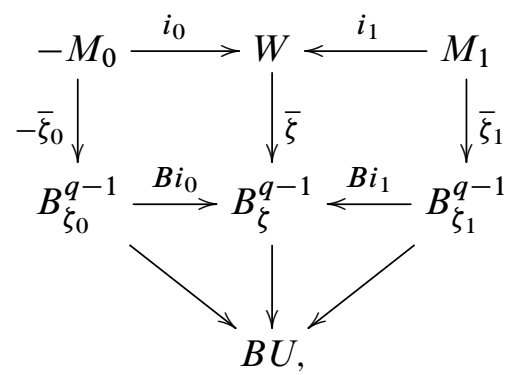

where $\bar{\zeta}_{0}, \bar{\zeta}_{1}$ and $\bar{\zeta}$ are all $(q-1)$-smoothings and for $j=0,1, i_{j}: M_{j} \rightarrow W$ are the inclusions and $B i_{j}$ are the corresponding induced maps of complex normal $(q-1)-$ types. Since $W$ is obtained from $M_{1}$ by the addition of handles on index $(q+1)$ or larger, the proof of [7, Lemma 2.9(3)] shows that $B i_{1}$ is an equivalence of complex normal $(q-1)$-types. We then set $\alpha$ to be the following map of fibrations over $B U$ :

$$
\alpha:=\left(B i_{1}\right)^{-1} \circ B i_{0}: B_{\zeta_{0}}^{q-1} \rightarrow B_{\zeta_{1}}^{q-1}
$$

where $\left(B i_{1}\right)^{-1}$ denotes a homotopy inverse of $B i_{1}$. By definition, $(W, \bar{\zeta})$ is a $\left(B_{\zeta_{1}}^{q-1}, \eta_{\zeta_{1}}^{q-1}\right)$-bordism which gives

$$
\alpha_{*}\left(\left[M_{0}, \bar{\zeta}_{0}\right]\right)=\left[M_{1}, \bar{\zeta}_{1}\right]
$$

Remark 2.15 Notice that [7, Theorem 3.8] is a direct consequence of the above result (by taking $\alpha=\mathrm{id}$ ). While [7, Theorem 3.8] is symmetric for $\left(M_{0}, \zeta_{0}\right)$ and $\left(M_{1}, \zeta_{1}\right)$, in Theorem 2.14 the direction of the map $\alpha$ breaks this symmetry. The above extension of our earlier result was suggested by a question from Andy Wand in Nantes in September 2013. 


\section{Maximal almost contact manifolds}

In dimension three the Stein cobordism relation has several interesting properties, one of which is that there are initial elements: There exists a contact manifold $\left(M_{\min }, \xi_{\min }\right)$ such that for any other contact 3-manifold $(M, \xi)$ we have

$$
\left(M_{\min }, \xi_{\min }\right) \prec(M, \xi) .
$$

In fact, by [12] any overtwisted contact structure on any manifold will do (see also [16]). On the other hand, in high dimensions, ie for $\operatorname{dim}(M)=2 q+1 \geq 5$ there exist final almost contact elements. It is not clear whether such objects exist in dimension three.

The next proposition provides a proof of Theorem 1.2 from Section 1.

Proposition 3.1 In every dimension $2 q+1 \geq 5$ there exists an almost contact manifold $\left(M_{\max }, \varphi_{\max }\right)$ so that for any almost contact manifold $(M, \varphi)$ we have

$$
(M, \varphi) \prec\left(M_{\max }, \varphi_{\max }\right) .
$$

Moreover, in dimensions 5 and 7 we can take certain almost contact structures on the nontrivial sphere bundles over $S^{2}$ as final elements:

$$
\left(M_{\max }, \varphi_{\max }\right)=\left(S^{3} \tilde{\times} S^{2}, \varphi_{\max }\right), \quad\left(M_{\max }, \varphi_{\max }\right)=\left(S^{5} \tilde{\times} S^{2}, \varphi_{\max }\right),
$$

where $\varphi_{\max }$ is any almost contact structure whose first Chern class is primitive.

Proof For $\left(M_{\max }, \varphi_{\max }\right)$ we can take any almost contact $(2 q+1)$-manifold where the corresponding stable complex manifold $\left(M_{\max }, \zeta_{\max }\right)$ has complex normal $(q-1)$-type $B_{\zeta_{\max }}^{q-1}=B U$ and the map to $B U$ is just the identity. To construct such a manifold, we begin with any stably complex $(2 q+1)$-manifold $\zeta: M \rightarrow B U$ and apply surgery below the middle dimension [7, Proposition 2.6] to obtain a stably complex manifold $\left(M_{\max }, \zeta_{\max }\right)$ where $\zeta_{\max }: M_{\max } \rightarrow B U$ is a $q$-equivalence, which then has the desired complex normal $(q-1)$-type. We then take $\varphi_{\max }$, to be any almost contact structure which stabilizes to $\zeta_{\max }$, which exists by [7, Lemma 2.17].

Now let $(M, \varphi)$ be any almost contact $(2 q+1)$-manifold with stable complex structure $\zeta$, complex normal $(q-1)$-type $\left(B_{\zeta}^{q-1}, \eta_{\zeta}^{q-1}\right)$ and with $\zeta$-compatible normal $(q-1)-$ smoothing $\bar{\zeta}: M \rightarrow B_{\zeta}^{q-1}$. By definition, $\eta_{\zeta}^{q-1}: B_{\zeta}^{q-1} \rightarrow B U$ is a fibration and we set $\alpha=\eta_{\zeta}^{q-1}$. Bordism of $B U$-manifolds is just ordinary complex bordism, and by [30, page 117] the odd bordism groups $\Omega_{2 q+1}^{U}$ are trivial, which implies that $\alpha_{*}([M, \bar{\zeta}])=$ $0=\left[M_{\max }, \zeta_{\max }\right]$. By Theorem 2.14 it follows that $(M, \zeta) \prec\left(M_{\max }, \zeta_{\max }\right)$ and due to the equivalence given in (1) above we finally conclude that $(M, \varphi) \prec\left(M_{\max }, \varphi_{\max }\right)$. 
In dimensions 5,7 one checks that the explicit manifolds stated in the proposition have the correct complex normal 1 - resp. 2-types. For this, note that $\pi_{1}(B U)=$ $\pi_{3}(B U)=0, \pi_{2}(B U)=\mathbb{Z}$ and the assumption that $c_{1}$ is primitive ensures that the second homotopy group of the associated type is $\mathbb{Z}$.

Remark 3.2 The almost contact manifold $\left(M_{\max }, \varphi_{\max }\right)$ is far from being unique. Indeed, if $\left(M_{\max }, \varphi_{\max }\right) \prec\left(M^{\prime}, \varphi^{\prime}\right)$ then $\left(M^{\prime}, \varphi^{\prime}\right)$ is also maximal for the topological Stein cobordism relation. For example, for any Stein fillable almost contact manifold $\left(M_{0}, \varphi_{0}\right),\left(M_{\max } \# M_{0}, \varphi_{\max } \# \varphi_{0}\right)$ is also maximal. Note also that $\left(M_{\max }, \varphi_{\max }\right)$ is necessarily Stein fillable (that is, contains a Stein fillable contact structure), shown by the Stein cobordism from, say, the standard contact sphere to $\left(M_{\max }, \varphi_{\max }\right)$.

Remark 3.3 At the level of contact structures, it seems very unlikely that the analogue of Proposition 3.1 holds. Specifically, it seems unlikely that there is a single contact $(2 n+1)$-manifold $\left(M_{\max }, \xi_{\max }\right)$ such that for every contact $(2 n+1)$-manifold $(M, \xi)$ the manifold $\left(M_{\max }, \xi_{\max }\right)$ is the out-going end of some Stein cobordism starting from $(M, \xi)$. However by Theorem 2.6 we have the following: For any maximal almost contact manifold $\left(M_{\max }, \varphi_{\max }\right)$ of dimension $(2 q+1) \geq 5$, and for any contact $(2 q+1)$-manifold $(M, \xi),\left(M_{\max }, \varphi_{\max }\right)$ admits a contact structure $\xi_{(M, \xi)}$ and a Stein cobordism from $(M, \xi)$ to $\left(M_{\max }, \xi_{(M, \xi)}\right)$.

A further interesting special case of the Stein cobordism relation occurs for "Calabi-Yau" almost contact structures on 5-manifolds and 7-manifolds.

Proposition 3.4 Let $n=5$ or 7 and let $(M, \varphi)$ be an almost contact $n$-manifold such that $c_{1}(\varphi)=0$. Then

$$
(M, \varphi) \prec\left(S^{n}, \varphi_{\text {std }}\right),
$$

where $\varphi_{\text {std }}$ denotes the almost contact structure underlying the standard contact structure on $S^{n}$ and the Stein cobordism $(W, J)$ can be assumed to have $c_{1}(J)=0$.

Proof Let $n=2 q+1$, so that $q=2$ or 3 and let $\zeta$ be the stabilization of the almost contact structure $\varphi$. Since $c_{1}(\varphi)=0$, the complex normal $(q-1)$-type of $(M, \zeta)$ factors through $B S U \rightarrow B U$ by [7, Lemma 2.22(ii), Lemma 2.23]. Since the complex normal $(q-1)$-type of $\left(S^{n}, \varphi_{\text {std }}\right)$ is $B S U \rightarrow B U$, the lemma follows from Theorem 2.14 and the fact that $\Omega_{5}^{\mathrm{SU}}=\Omega_{7}^{\mathrm{SU}}=0$; see [30, page 248].

Remark 3.5 In contrast to the 3-dimensional case, there can be no minimal elements with respect to the Stein cobordism relation in dimension at least 5. For example if $\left(M_{0}, \varphi_{0}\right) \prec\left(M_{1}, \varphi_{1}\right)$ and $c_{1}\left(\varphi_{1}\right)=0$, then the fact that $c_{1}\left(\varphi_{1}\right)=0$ implies that 
$c_{1}\left(\varphi_{0}\right)=0$. To see this note that a topological Stein cobordism $(W, J)$ from $M_{0}$ to $M_{1}$ is obtained from $M_{1}$ by attaching high index handles, which means that $c_{1}(J)=0$. But this would imply that an initial element $\left(M_{\min }, \varphi_{\min }\right)$ must have $c_{1}\left(\varphi_{\min }\right)=0$. A similar argument shows that for certain choices of $M_{1}$ the fact that $c_{1}^{q}\left(\varphi_{1}\right) \neq 0$ implies $c_{1}^{q}\left(\varphi_{\min }\right) \neq 0\left(\operatorname{cf}\left[7\right.\right.$, Proposition 6.2]). For this note that the inclusion of $M_{1}$ into $W$ gives an isomorphism on fundamental groups. Suppose that $\beta \in H^{1}\left(B \pi_{1}(W)\right)$ is a class such that when restricted to $M_{1}$

$$
p_{1}^{*}(\beta) \cup c_{1}^{q}(J) \neq 0,
$$

where $p_{1}: W \rightarrow B \pi_{1}(W)$ is the classifying map of the universal cover of $W$. It follows that the restriction of $p_{1}^{*}(\beta) \cup c_{1}^{q}(J) \neq 0$ to $M_{0}=M_{\min }$ is also nonzero and hence that $c_{1}\left(\varphi_{\min }\right)=\left.c_{1}(J)\right|_{M_{\min }} \neq 0$.

In fact, these sorts of arguments show that there can be no initial elements even if one forgets about the almost contact structures, and one simply considers Pontryagin classes rather than Chern classes.

\section{Stein fillability and connected sums}

The connected sum of Stein fillable manifolds is again Stein fillable, since adding a one-handle to the Stein fillings can be done in a way that is compatible with Stein structures. Eliashberg [10, Section 8] has shown that the converse of this statement holds for 3-manifolds: a connected sum of 3-manifolds is Stein fillable if and only if both summands are. In addition, the Stein fillable structures on the components can be chosen so that their connected sum is isotopic to the given Stein fillable structure on the connected sum. (For a detailed description of the contact connected sum construction, see [15, pages 301-302].) Extensions of some aspects of the above result of Eliashberg to higher dimensions are given in [17].

In this section we prove Theorem 1.5, which shows that in higher dimensions the summands of a Stein fillable almost contact manifold in a connected sum decomposition are not necessarily Stein fillable. To make this statement precise, we note that the summands are well-defined only up to almost diffeomorphism, that is, up to connected sum with homotopy spheres. Indeed, for $M_{1} \# M_{2}$ the manifolds $M_{1}-$ int $D^{n}$ and $M_{2}$-int $D^{n}$ can be turned into closed manifolds (by gluing back $D^{n}$ ) in many different ways, differing by connected sums with homotopy spheres. Below we find examples of almost contact connected sums where the summands are not Stein fillable, even after the addition of homotopy spheres.

For the proof of Theorem 1.5 we examine the Stein fillability of certain almost contact structures on the unit cotangent bundle $S T^{*} S^{2 k+1}$ of the $(2 k+1)$-sphere $S^{2 k+1}$. We 
first need to establish some preliminary results. Lemma 4.1 is a small elaboration of a theorem of Milnor and Spanier about the topology of $S T^{*} S^{2 k+1}$. Proposition 4.2 gives a description of the topology of possible Stein fillings of manifolds almost diffeomorphic to $S T^{*} S^{2 k+1}$, which may be of independent interest. Finally, Lemmas 4.4 and 4.5 show that $S T^{*} S^{2 k+1}$ admits an almost contact structure $\varphi$ which is not Stein fillable, provided $k \geq 5$ is odd.

Lemma 4.1 (cf [26, Theorem 2]) There is a map $f: S T^{*} S^{2 k+1} \rightarrow S^{2 k}$ such that the induced homomorphism $f_{*}: H_{2 k}\left(S T^{*} S^{2 k+1}\right) \rightarrow H_{2 k}\left(S^{2 k}\right)$ is an isomorphism if and only if $k=0,1$ or 3 .

Proof Let $\pi: S T^{*} S^{2 k+1} \rightarrow S^{2 k+1}$ be the bundle projection of the unit cotangent bundle of $S^{2 k+1}$. If we consider $\pi$ merely as a spherical fibration, then a map $f$ as in the statement of the lemma exists if and only if $\pi$ is trivial as a spherical fibration. This is because the product map

$$
f \times \pi: S T^{*} S^{2 k+1} \rightarrow S^{2 k} \times S^{2 k+1}
$$

is a homology isomorphism, and so by Whitehead's theorem a homotopy equivalence, and this gives a fiber homotopy trivialization of $\pi$. By [26, Theorem 2], the bundle projection $\pi$ is trivial as a spherical fibration if and only if $k=0,1$ or 3 .

The proof of the following proposition uses handle canceling and the Whitney trick, which are familiar from the proof of the $h$-cobordism theorem in higher dimensions. For details concerning these constructions we refer to $[25 ; 33]$.

Proposition 4.2 Let $k \geq 1$ and let $M=\left(S T^{*} S^{2 k+1}\right) \# \Sigma_{0}$ be the connected sum of $S T^{*} S^{2 k+1}$ with some homotopy sphere $\Sigma_{0}$. Choose some almost contact structure $\varphi$ on $M$ and let $W^{4 k+2}$ be the smooth manifold underlying a Stein filling of $(M, \varphi)$. Then $W$ decomposes as a boundary connected sum

$$
W=W_{l} \curvearrowleft W_{\Sigma},
$$

where $H_{2 k}\left(W_{l}\right)=\mathbb{Z} / l \mathbb{Z}$ and $W_{\Sigma}$ is a $2 k$-connected filling of some homotopy sphere $\Sigma$. Moreover we have the following possibilities for the topology of $W_{l}$ :

(1) If $l>1$, then $W_{l}$ has a handle decomposition with precisely one handle of index $2 k$ and two handles of index $2 k+1$.

(2) If $l=1$, then $W_{1} \cong D T^{*} S^{2 k+1}$.

(3) If $l=0$, then we must have $k=1,3$ and $W_{0} \cong D^{2 k+2} \times S^{2 k}$.

In particular, $S T^{*} S^{2 k+1}$ admits a subcritical filling if and only if $k=1,3$. 
Proof First note that $M=\partial W$ is $(2 k-1)-$ connected and that

$$
H_{*}(\partial W) \cong H_{*}\left(S^{2 k} \times S^{2 k+1}\right) .
$$

It follows that any Stein filling of $M$ is $(2 k-1)$-connected and hence by the Hurewicz theorem the map $\pi_{2 k+1}(W) \rightarrow H_{2 k+1}(W)$ is surjective. The long exact sequence of the pair

$$
H_{2 k+1}(\partial W) \stackrel{\iota}{\longrightarrow} H_{2 k+1}(W) \longrightarrow H_{2 k+1}(W, \partial W) \longrightarrow H_{2 k}(\partial W) \longrightarrow H_{2 k}(W) \longrightarrow 0
$$

yields that $H_{2 k}(W)=\mathbb{Z} / l \mathbb{Z}$ is cyclic and we also know that $H_{2 k+1}(W)$ is torsion free since $W$ admits a handle decomposition without handles of index greater than $2 k+2$ by assumption. Moreover, the intersection pairing is unimodular on a complement $H$ to $\operatorname{im}(\iota) \subseteq H_{2 k+1}(W)$. We let $\left\{x_{1}, \ldots, x_{2 r}\right\}$ be a symplectic basis for $H$ consisting of primitive elements, which can in turn be represented by spheres. Since the target is simply connected, we can use the Whitney trick to find embedded representatives in the interior of $W$. We can furthermore assume that the geometric intersection numbers of these spheres agree with their algebraic intersection numbers.

Thus we have a configuration of embedded $(2 k+1)$-dimensional spheres $\left\{S_{1}, \ldots, S_{2 r}\right\}$ having a regular neighborhood $N$ whose boundary is a homotopy sphere $\Sigma$. It follows that $W$ decomposes as a boundary connected sum

$$
W=W_{l} \curvearrowleft W_{\Sigma},
$$

where $W_{\Sigma} \cong N$ is $2 k$-connected. This boundary connected sum is obtained by choosing an embedded path $\gamma$ from $\partial N$ to $\partial W$ and removing $N$ along with a tubular neighborhood of $\gamma$. Note that in this decomposition $\partial W_{l}=M \#(-\Sigma)$. Applying Mayer-Vietoris, we conclude that $H_{2 k}\left(W_{l}\right) \cong \mathbb{Z} / l \mathbb{Z}$. This proves the first part of the proposition.

We now consider the topology of $W_{l}$ and prove the remainder of the proposition. $W_{l}$ is $(2 k-1)$-connected, hence it follows by handle canceling that $W_{l}$ is obtained by attaching handles of index at least $2 k$ to $S T^{*} S^{2 k+1} \# \Sigma_{0} \#(-\Sigma)$. Turning this handle decomposition upside down gives a handle decomposition with handles of index at most $2 k+2$. Then by further cancellation of handles we can find a handle decomposition with at most one handle of index $2 k$ and at most two handles of index $2 k+1$, where we use the fact that $W_{l}$ is simply connected. This proves case (1).

If $l=1$, then the handle decomposition of $W_{l}$ reduces further to contain a single $(2 k+1)$-handle, and $W_{1}$ is diffeomorphic to a linear $D^{2 k+1}$-bundle over $S^{2 k+1}$. This bundle must be stably trivial, otherwise $M$ will have a nontrivial stable tangent 
bundle. Moreover, by analyzing the homotopy long exact sequence of the fibration

$$
\mathrm{SO}(2 k+1) \rightarrow \mathrm{SO}(2 k+2) \rightarrow S^{2 k+1},
$$

one sees that any stably trivial bundle over $S^{2 k+1}$ is either trivial or isomorphic to the unit tangent bundle of the sphere. If $k \neq 1,3$ then $W_{1}$ cannot be the total space of the trivial bundle, because this would give rise to the existence of a map $f: M \rightarrow S^{2 k}$ as in Lemma 4.1 which is impossible. On the other hand, if $k=1,3$, then the tangent bundle of $S^{2 k+1}$ is trivial. In both cases we conclude that there is a diffeomorphism $W_{1} \cong D T^{*} S^{2 k+1}$, which proves case (2).

If $l=0$, then $H_{2 k}(W)=H_{2 k}\left(W_{0}\right)=\mathbb{Z}$. It follows that the handle decomposition of $W_{0}$ has just one $2 k$-handle and hence $W_{0}$ is diffeomorphic to a linear $D^{2 k+2}$-bundle over $S^{2 k}$. This bundle is stable and must be stably trivial, otherwise the tangent bundle of $M$ would not be stably trivial. We conclude that $W_{0}$ is diffeomorphic to $D^{2 k+2} \times S^{2 k}$.

Remark 4.3 Although not the focus of this work, the topology of Stein fillings, as opposed to their boundaries, is of independent interest and may be relevant to certain computations in contact homology. In the case that $k=1$, Proposition 4.2 determines the smooth manifolds underlying Stein fillings of almost contact structures on $S^{2} \times S^{3}$. In this dimension there are no exotic 5-spheres [22] and by [29, Theorem 6.2] the manifold $W_{\Sigma}$ is diffeomorphic to $\#{ }_{r}\left(S^{3} \times S^{3}\right)-\operatorname{Int}\left(D^{6}\right)$. Concerning the manifolds $W_{l}$, we conjecture that they are classified up to diffeomorphism by $l$. If this is correct, then topological Stein fillings $(W, J)$ of $S^{2} \times S^{3}$ are classified up to stably complex diffeomorphism by their integral homology groups $H_{*}(W)$ along with their first Chern class $c_{1}(J) \in H^{2}(W)$.

Lemma 4.4 If $k$ is odd, there exist almost contact structures $\varphi$ on $S T^{*} S^{2 k+1}$ with nonzero $k^{\text {th }}$ Chern class, $0 \neq c_{k}(\varphi) \in H^{2 k}\left(S T^{*} S^{2 k+1}\right) \cong \mathbb{Z}$.

Proof Let $\varphi_{\text {can }}$ be the standard almost contact structure underlying the canonical contact structure on $M=S T^{*} S^{2 k+1}$, and let $\zeta_{\text {can }}$ be the stable complex structure determined by $\varphi_{\text {can }}$. Since $\zeta_{\text {can }}$ extends over $D T^{*} S^{2 k+1}$, we have that $c_{k}\left(\varphi_{\text {can }}\right)=$ $c_{k}\left(\zeta_{\text {can }}\right)=0$. To find a stable complex structure $\zeta$ with $c_{k}(\zeta) \neq 0$, we recall that the group $[M, \mathrm{SO} / U]$ acts freely and transitively on the set of homotopy classes of stable complex structures on $M$. Now if we let $M^{\bullet}:=M \backslash B^{4 k+1}$ be the manifold obtained by removing a ball, then there is a homotopy equivalence $M^{\bullet} \simeq S^{2 k} \vee S^{2 k+1}$ and hence

$$
\left[M^{\bullet}, \mathrm{SO} / U\right] \cong \pi_{2 k}(\mathrm{SO} / U) \oplus \pi_{2 k+1}(\mathrm{SO} / U)
$$


Since $k$ is odd, $\pi_{4 k}(\mathrm{SO} / U)=0$ [3], and so there is no obstruction to extending a map $M^{\bullet} \rightarrow \mathrm{SO} / U$ to a map $M \rightarrow \mathrm{SO} / U$. Hence the restriction map $[M, \mathrm{SO} / U] \rightarrow$ $\left[M^{\bullet}, \mathrm{SO} / U\right]$ is onto. Again using that $k$ is odd, the boundary map, $\pi_{2 k}(\mathrm{SO} / U) \rightarrow$ $\pi_{2 k-1}(U)$, in the homotopy long exact sequence of the fibration $U \rightarrow \mathrm{SO} \rightarrow \mathrm{SO} / U$ is nonzero [3]. Since $\pi_{2 k-1}(U)$ classifies stable unitary bundles over $S^{2 k}$ which are in turn classified by their $k^{\text {th }}$ Chern class [19, Proposition 9.1], it follows from (3) and the discussion above that we can choose $\psi \in[M, \mathrm{SO} / U]$ such that $\zeta:=\zeta_{\text {can }}+\psi$ has $c_{k}(\zeta) \neq 0$. By [7, Lemma 2.17], we know that $\zeta$ destabilizes to an almost contact structure $\varphi$, which then also has $c_{k}(\varphi) \neq 0$.

Lemma 4.5 Let $\varphi$ be an almost contact structure on $M=S T^{*} S^{2 k+1}$ with $c_{k}(\varphi) \neq 0$ and let $\left(\Sigma, \varphi_{\Sigma}\right)$ be any almost contact homotopy sphere. If $k \neq 1,3$, then neither $\left(M \# \Sigma, \varphi \# \varphi_{\Sigma}\right)$ nor $\left(-(M \# \Sigma),-\left(\varphi \# \varphi_{\Sigma}\right)\right)$ is Stein fillable.

Proof Suppose that $(W, J)$ is a Stein filling of $\left(M \# \Sigma, \varphi \# \varphi_{\Sigma}\right)$. Since $c_{k}\left(\varphi \# \varphi_{\Sigma}\right)=$ $c_{k}(\varphi)$ is nonzero and pulls back from $c_{k}(J) \in H^{2 k}(W)$, we conclude that $H^{2 k}(W)$ is infinite. Now by Proposition 4.2, this can only happen if $k=1,3$. Since we assumed $k \neq 1,3$, no such Stein filling $(W, J)$ can exist. For the reversed orientation, we use [7, Propostion 6.7] which states that $\left(M \# \Sigma, \varphi \# \varphi_{\Sigma}\right)$ is Stein fillable if and only if $\left(-(M \# \Sigma),-\left(\varphi \# \varphi_{\Sigma}\right)\right)$ is Stein fillable.

Proof of Theorem 1.5 We let $k \geq 5$ be odd and set $(M, \varphi)=\left(S T^{*} S^{2 k+1}, \varphi\right)$, where we have $c_{k}(\varphi) \neq 0$. By Lemma 4.4 such almost contact structures always exist. Now, by Lemma 4.5 , for any almost contact homotopy sphere $\left(\Sigma, \varphi_{\Sigma}\right)$ neither $\left(M \# \Sigma, \varphi \# \varphi_{\Sigma}\right)$ nor $\left(-(M \# \Sigma),-\left(\varphi \# \varphi_{\Sigma}\right)\right)$ is Stein fillable.

On the other hand, we let $M^{\bullet}=M \backslash B^{4 k+1}$ and we let $\varphi^{\bullet}$ be the induced almost contact structure on $M^{\bullet}$. We set $W^{4 k+2}:=M^{\bullet} \times[0,1]$, which has a natural almost complex structure $\varphi^{\bullet} \times[0,1]$ induced by $\varphi^{\bullet}$. Moreover, the smoothened boundary of $W$ with the induced almost contact structure is precisely $(M, \varphi) \#(-M,-\varphi)$. Since $M$ is $(2 k-1)$-connected, it follows from a theorem of Smale [28, Theorem C], that $M^{\bullet}$ admits a handle decomposition with handles of index less than or equal to $2 k+1$. Since any handle decomposition on $M^{\bullet}$ gives rise to one on $M^{\bullet} \times I$ with handles of the same index, we have that $W=M^{\bullet} \times[0,1]$ admits a handle decomposition with handles of index less than or equal to $2 k+1$. Consequently $(W, \varphi \times[0,1])$ is a topological Stein filling of $(M, \varphi) \#(-M,-\varphi)$, which then admits a Stein fillable contact representative $\xi_{M \#(-M)}$ by Theorem 2.4 .

Remark 4.6 Notice that the nonfillability of $\left(S T^{*} S^{2 k+1}, \varphi\right)$ in Theorem 1.5 arises from the choice of the almost contact structure $\varphi$, since $S T^{*} S^{2 k+1}$ does admit Stein 
fillable contact structures. In [5] we shall prove a stronger version of Theorem 1.5 which asserts the existence of $(4 k-1)$-connected closed smooth $(8 k+1)$-manifolds $M$, such that $M$ (and $M \# \Sigma$ for any homotopy sphere $\Sigma$ ) admits no Stein fillable almost contact structure at all, but $M \#(-M)$ is Stein fillable. We would like to point out that our result is on the almost contact level: we do not claim that the Stein fillable contact structure $\xi_{M \#(-M)}$ on $M \#(-M)$ found in the proof of Theorem 1.5 (representing the almost contact structure $\varphi \#(-\varphi)$ ) can be given as a connected sum $\xi_{+} \# \xi_{-}$where $\xi_{ \pm}$is a contact structure on $\pm M$.

We now turn to dimension 5 and prove Theorem 1.6, restated below as Theorem 4.8. Notice that in dimension five the connected sum $M_{0} \# M_{1}$ determines the diffeomorphism type of its components $M_{0}$ and $M_{1}$, since there are no exotic 5-spheres. The following lemma extends this statement to almost contact 5-manifolds.

Lemma 4.7 Let $\varphi$ be an almost contact structure on the connected sum of 5-manifolds $M_{0}$ and $M_{1}$. Then there are, up to homotopy unique, almost contact structures $\varphi_{0}$ on $M_{0}$ and $\varphi_{1}$ on $M_{1}$, such that $\left(M_{0} \# M_{1}, \varphi\right)=\left(M_{0} \# M_{1}, \varphi_{0} \# \varphi_{1}\right)$.

Proof For $i=0,1$, let $M_{i}^{\bullet}:=M_{i}-\operatorname{int}\left(D^{5}\right) \subset M_{0} \# M_{1}$ be the punctured copy of $M_{i}$ contained in the connected sum. We define $\left.\varphi_{i}\right|_{M_{i}^{\bullet}}:=\left.\varphi\right|_{M_{i}^{\bullet}}$. It remains to show that there is a unique extension of $\left.\varphi_{i}\right|_{M_{i}^{\bullet}}$ to an almost contact structure on $M_{i}$. Now the obstruction to extension lies in $\pi_{4}(\mathrm{SO}(5) / U(2))$ and the obstruction to uniqueness lies in $\pi_{5}(\operatorname{SO}(5) / U(2))$. By [23], we have $\pi_{4}(\operatorname{SO}(5) / U(2))=\pi_{5}(\operatorname{SO}(5) / U(2))=0$, which concludes the proof.

With the aid of this lemma we have the following theorem.

Theorem 4.8 Let $(M, \varphi)=\left(M_{0} \# M_{1}, \varphi_{0} \# \varphi_{1}\right)$ be a Stein fillable almost contact 5 -manifold. Assume that either $c_{1}(\varphi)=0$ or that

$$
c_{1}(\varphi)\left(\pi_{2}\left(M_{0}\right)\right)=c_{1}(\varphi)\left(\pi_{2}\left(M_{1}\right)\right)=\mathbb{Z}=\pi_{2}(B U) .
$$

Then both $\left(M_{0}, \varphi_{0}\right)$ and $\left(M_{1}, \varphi_{1}\right)$ are Stein fillable.

Proof Let $\zeta, \zeta_{0}$ and $\zeta_{1}$ be the stable complex structures determined by $\varphi, \varphi_{0}$ and $\varphi_{1}$ respectively. After stabilizing we have $(M, \zeta)=\left(M_{0}, \zeta_{0}\right) \#\left(M_{1}, \zeta_{1}\right)$. Also, let $K_{i}=K\left(\pi_{1}\left(M_{i}\right), 1\right)$ so that $K\left(\pi_{1}(M), 1\right)=K_{0} \vee K_{1}$. Under the assumptions of the proposition, [7, Lemma 2.13] implies that the complex normal 1-type of $(M, \zeta)$ is given by

$$
\left(B_{\zeta}^{1}, \eta_{\zeta}^{1}\right) \cong\left(B \mathrm{SU} \times\left(K_{0} \vee K_{1}\right), \mathrm{pr}_{B \mathrm{SU}}\right)
$$


if $c_{1}(\varphi)=0$ and

$$
\left(B_{\zeta}^{1}, \eta_{\zeta}^{1}\right) \cong\left(B U \times\left(K_{0} \vee K_{1}\right), \operatorname{pr}_{B U}\right)
$$

if $c_{1}(\varphi)\left(\pi_{2}\left(M_{0}\right)\right)=c_{1}(\varphi)\left(\pi_{2}\left(M_{1}\right)\right)=\pi_{2}(B U)=\mathbb{Z}$. Since $\eta_{\zeta}^{1}$ is the projection to $B \mathrm{SU}$ resp. $B U$, there is a canonical isomorphism $\Omega_{5}\left(B_{\zeta}^{1} ; \eta_{\zeta}^{1}\right) \cong \Omega_{5}^{G}\left(K_{0} \vee K_{1}\right)$ for $G=U$ or SU, which we use in the remainder of the proof.

Let us now assume that $c_{1}(\varphi)=0$. The argument in the other cases is formally the same, and is given by replacing the map $B \mathrm{SU} \rightarrow B U$ by Id: $B U \rightarrow B U$. Let $\bar{\zeta}: M \rightarrow B_{\zeta}^{1}$ be a $\zeta$-compatible normal 1 -smoothing. We first consider the product smoothing

$$
\bar{\zeta} \circ \operatorname{pr}_{M}: M \times[0,1] \rightarrow M \rightarrow B_{\zeta}^{1}
$$

and attach a 6-dimensional 5-handle to $M \times\{1\}$ along the connect sum locus

$$
S^{4} \times[0,1] \subset M=M_{0} \# M_{1}
$$

to obtain a bordism $W$. Since $\pi_{4}(\mathrm{SO} / \mathrm{SU})=0$, there is no obstruction to making this a stably complex 5-handle (see [7, Section 2.3]), and indeed there is no obstruction to extending $\bar{\zeta} \circ \mathrm{pr}_{M}$ to a normal smoothing $\bar{\zeta}_{W}^{\prime}: W \rightarrow B_{\zeta}^{1}$. Consider the map $\bar{\zeta}_{W}$ given by taking the composition of $\bar{\zeta}_{W}^{\prime}$ with the collapsing map induced by the wedge sum:

$$
W \stackrel{\bar{\zeta}_{W}^{\prime}}{\longrightarrow} B \mathrm{SU} \times\left(K_{0} \vee K_{1}\right) \stackrel{\text { col }}{\longrightarrow} B \mathrm{SU} \times K_{1} .
$$

This is a normal map, and setting $\bar{\zeta}_{i}$ to be the restriction of $\zeta_{W}$ to $M_{i}$ we see that the bordism $\left(W, \bar{\zeta}_{W}\right)$ gives the equality

$$
[M, \operatorname{col} \circ \bar{\zeta}]=\left[M_{0}, \bar{\zeta}_{0}\right]+\left[M_{1}, \bar{\zeta}_{1}\right] \in \Omega_{5}^{\mathrm{SU}}\left(K_{1}\right)
$$

Now, by Theorem 2.12, $[M, \bar{\zeta}]=0$ since $(M, \varphi)$ is Stein fillable, and consequently the bordism class $[M, \mathrm{col} \circ \bar{\zeta}]$ is trivial too. Moreover, since the composition

$$
\operatorname{pr}_{K_{1}} \circ \bar{\zeta}_{0}: M_{0} \rightarrow K_{1}
$$

is null-homotopic and the bordism group $\Omega_{5}^{\mathrm{SU}}=\Omega_{5}^{U}=0$ according to [30, page 248], it follows that $\left[M_{0}, \bar{\zeta}_{0}\right]=0$. Hence the bordism class $\left[M_{1}, \bar{\zeta}_{1}\right]$ is trivial. Since the map

$$
\bar{\zeta}_{1}: M_{1} \rightarrow B \mathrm{SU} \times K_{1}
$$

is a $\zeta_{1}$-compatible normal 1 -smoothing, Theorem 2.12 implies that $\left(M_{1}, \varphi_{1}\right)$ is Stein fillable. The same argument mutatis mutandis shows that $\left(M_{0}, \varphi_{0}\right)$ is Stein fillable as well.

Remark 4.9 We point out that in dimension five the method in the proof of Theorem 4.8 does not ascend to give control over contact structures. That is, if the almost contact 
manifolds $\left(M_{0}, \varphi_{0}\right)$ and $\left(M_{1}, \varphi_{1}\right)$ are induced from contact manifolds $\left(M_{0}, \xi_{0}\right)$ and $\left(M_{1}, \xi_{1}\right)$, and even if we know that $\left(M_{0} \# M_{1}, \xi_{0} \# \xi_{1}\right)$ is Stein fillable, then in contrast to the situation in dimension 3 , we cannot conclude that $\left(M_{0}, \xi_{0}\right)$ and $\left(M_{1}, \xi_{1}\right)$ are Stein fillable.

Remark 4.10 Note that in the proof of Theorem 4.8 involved constructing a nullbordism of each component of the connected sum $M_{0} \# M_{1}$ by first adding a 5-handle and then capping off two of the resulting boundary components. This bordism is thus far from having the correct homotopy type, but applying surgery below the middle dimension as in the proof of Theorem 2.12 has the virtue of remedying this.

\section{Nonfillable almost contact structures on highly connected manifolds}

In dimensions congruent to $7 \bmod 8$, the isomorphism $\pi_{8 k-1}(\mathrm{SO} / U) \cong \mathbb{Z}_{2}$ means that there are precisely two homotopy classes of stable almost contact structures on $S^{8 k-1}$. One of these homotopy classes, denoted $\zeta_{\text {std }}$, bounds over $D^{8 k}$ and is thus Stein fillable. Let us call the other stable almost contact structure on $S^{8 k-1}$ exotic and denote it by $\zeta_{\mathrm{ex}}$. By [7, Theorem 1.3] we know that $\left(S^{7}, \zeta_{\mathrm{ex}}\right)$ is Stein fillable. Indeed, (according to Theorem 5.7 below) the quaternionic projective plane $\mathbb{H} \mathbb{P}^{2}$ admits no almost complex structure, but if we puncture it, then as the Hopf $D^{4}$-bundle over $S^{4}$ it does. Thus the punctured $\mathbb{H} \mathbb{P}^{2}$ provides a filling of $\left(S^{7}, \zeta_{\mathrm{ex}}\right)$ which admits a Stein structure, inducing a Stein fillable contact structure on $S^{7}$ that stabilizes to $\zeta_{\text {ex }}$. In higher dimensions, however, we have the following result (which corresponds to Theorem 1.7 from Section 1):

Theorem 5.1 The exotic stable complex structure $\zeta_{\text {ex }}$ on $S^{8 k-1}$ cannot be represented by a Stein fillable contact structure once $k \geq 2$.

Before giving the proof of this result, we derive Corollary 1.9 as a simple consequence.

Proof of Corollary 1.9 Let $\varphi$ be an almost contact structure on the (4k-2)-connected oriented $(8 k-1)$-manifold $M$. If $(M, \varphi)$ is not Stein fillable, we are done. If $(M, \varphi)$ is Stein fillable, let $\zeta$ be the stable complex structure determined by $\varphi$ and observe that the complex normal $(4 k-2)$-type of $(M, \zeta)$ is $\left(B U\langle 4 k\rangle, \pi_{4 k}\right)$ from Example 2.11, with associated bordism groups $\Omega_{*}^{U\langle 4 k-1\rangle}$. Let

$$
\bar{\zeta}: M \rightarrow B_{\zeta}^{4 k-2}
$$


be a (4k-2)-smoothing. By Theorem 2.12 we have $[M, \bar{\zeta}]=0 \in \Omega_{8 k-1}^{U\langle 4 k-1\rangle}$. The connected sum $\left(M, \bar{\zeta} \# \bar{\zeta}_{\mathrm{ex}}\right):=(M, \bar{\zeta}) \#\left(S^{8 k-1}, \bar{\zeta}_{\mathrm{ex}}\right)$ does not change the complex normal $(4 k-2)-$ type, and

$$
\left[M, \bar{\zeta} \# \bar{\zeta}_{\mathrm{ex}}\right]=[M, \bar{\zeta}]+\left[S^{8 k-1}, \bar{\zeta}_{\mathrm{ex}}\right] \in \Omega_{8 k-1}^{U\langle 4 k-1\rangle}
$$

By Theorem 5.1 the stable complex manifold $\left(S^{8 k-1}, \zeta_{\mathrm{ex}}\right)$ is not Stein fillable, and so by Theorem 2.12, $\left[S^{8 k-1}, \bar{\zeta}_{\mathrm{ex}}\right] \neq 0$, since $\left(B U\langle 4 k\rangle, \pi_{4 k}\right)$ is the complex normal $(4 k-2)-$ type of $\left(S^{8 k-1}, \bar{\zeta}_{\mathrm{ex}}\right)$. It follows that $\left[M, \bar{\zeta} \# \bar{\zeta}_{\mathrm{ex}}\right] \neq 0$ and consequently $\left(M, \zeta \# \zeta_{\mathrm{ex}}\right)$ is not Stein fillable by Theorem 2.12, since $\left(B U\langle 4 k\rangle, \pi_{4 k}\right)$ is the complex normal $(4 k-2)-$ type of $\left(M, \zeta \# \zeta_{\mathrm{ex}}\right)$.

Remark 5.2 According to [14, Proposition 6(vi)], the hypothesis of Corollary 1.9 that the $(4 k-2)-$ connected $(8 k-1)-$ manifold $M$ admit an almost contact structure is equivalent to assuming that $\operatorname{Im}\left(\tau_{M *}\right) \subseteq F_{*}\left(\pi_{4 k}(B U)\right)$. In Theorem 5.7(3) below, we prove that provided $k \geq 2$, the same condition is a necessary and sufficient condition for a $(4 k-1)-$ connected $8 k$-manifold to admit a stable complex structure.

Remark 5.3 The stable almost contact structures $\zeta$ and $\zeta \# \zeta_{\text {ex }}$ appearing in the proof of Corollary 1.9 differ by precisely the "top-dimensional $\mathbb{Z} / 2$-obstruction to stable homotopy of almost contact structures" identified by Geiges in [14, Theorem 4(2b)].

Remark 5.4 By [2] the stable almost contact structure found in Corollary 1.9 (as any stable complex, or even almost contact structure) can be represented by an overtwisted contact structure; according to Theorem 5.1 the stable almost contact structure $\zeta_{\text {ex }}$ on $S^{8 k-1}$ as well as the stable contact structure found by Corollary 1.9 cannot be represented by Stein fillable contact structures. It would be interesting to see if these particular stable complex structures admit fillable or tight (that is, not overtwisted) contact representatives.

\subsection{Almost complex $8 k$-manifolds}

In order to prove Theorem 5.1, we first improve a theorem of Yang [34] determining which smooth closed oriented $(4 k-1)$-connected $8 k$-manifolds admit stable complex structures.

Let $Y$ be a smooth closed oriented $(4 k-1)$-connected $8 k$-manifold, let $\tau_{Y}: Y \rightarrow B$ SO classify the stable tangent bundle of $Y$ and let

$$
\tau_{Y *}: \pi_{4 k}(Y) \rightarrow \pi_{4 k}(B \mathrm{SO})
$$


be the induced homomorphism. If $Y$ admits a stably complex structure, then $\tau_{Y}$ factors through $F: B U \rightarrow B$ SO and in this case $\operatorname{Im}\left(\tau_{Y *}\right) \subseteq F_{*}\left(\pi_{4 k}(B U)\right) \subseteq \pi_{4 k}(B S O)$. Now let $B_{i}$ denote the $i^{\text {th }}$ Bernoulli number (where we use the topological indexing and sign conventions, in particular, $B_{1}=\frac{1}{6}$ and $B_{2}=\frac{1}{30}$ ). The following theorem is a straightforward reformulation of (2) and (3) of [34, Theorem 1].

Theorem 5.5 (cf [34, Theorem 1(2),(3)]) A smooth closed oriented (4k-1)-connected $8 k$-manifold $Y$ with signature $\sigma_{Y}$ admits a stable complex structure if and only if:

(1) $k$ is odd and $\left(\frac{B_{2 k}+B_{k}}{B_{2 k} B_{k}} \cdot \frac{1}{2^{4 k-2}}\right) \sigma_{Y} \equiv 0 \bmod 2$.

(2) $k$ is even, $\operatorname{Im}\left(\tau_{Y *}\right) \subseteq F_{*}\left(\pi_{4 k}(B U)\right)$ and $\left(\frac{B_{2 k}-B_{k}}{B_{2 k} B_{k}} \cdot \frac{4 k}{2^{4 k}}\right) \sigma_{Y} \equiv 0 \bmod 2$.

Remark 5.6 Using the Hurewicz isomorphism $\pi_{4 k}(Y) \cong H_{4 k}(Y)$ and the universal coefficient theorem, we regard the homomorphism $\tau_{Y *}: \pi_{4 k}(Y) \rightarrow \pi_{4 k}(B \mathrm{SO})=\mathbb{Z}$ as a cohomology class $\tau_{Y *} \in H^{4 k}(Y)$, which Yang denotes by $v$. When $k$ is even, $F_{*}\left(\pi_{4 k}(B U)\right) \subset \pi_{4 k}(B S O)$ is the subgroup of index two [3], and so the condition $\operatorname{Im}\left(\tau_{Y *}\right) \subseteq F_{*}\left(\pi_{4 k}(B U)\right)$ is equivalent to the condition that $\tau_{Y *}$ vanishes mod 2, which is the condition Yang uses.

The following result, Theorem 1.8 from Section 1, simplifies Yang's theorem by removing the assumptions involving Bernoulli numbers from its statement.

Theorem 5.7 A smooth closed oriented (4k-1)-connected 8k-manifold $Y$ admits a stable almost complex structure if and only if:

(1) $k \geq 3$ is odd.

(2) $k=1$ and the signature $\sigma_{Y}$ of $Y$ is even.

(3) $k$ is even and $\operatorname{Im}\left(\tau_{Y *}\right) \subseteq F_{*}\left(\pi_{4 k}(B U)\right)$.

Remark 5.8 The simplification achieved in moving from Theorem 5.5 to Theorem 5.7 is perhaps surprising and rests on Theorem A.1, which is a nontrivial fact about the differences of reciprocals of Bernoulli numbers. Theorem 5.7 can be interpreted as a statement about the characteristic numbers (signature and $p_{k}^{2}$ ) of closed $(4 k-1)-$ connected almost complex smooth manifolds and the bordism groups $\Omega_{8 k}^{U\langle 4 k-1\rangle}$. It would be interesting to see if there are further connections between number theory and the characteristic numbers of closed $j$-connected almost complex $n$-manifolds for other values of $j$ and $n$. 
Proof of Theorem 5.7 For $k=1,2, B_{1}=\frac{1}{6}$ and $B_{2}=B_{4}=\frac{1}{30}$, see eg [18, page 12]. Hence

$$
\frac{B_{2}+B_{1}}{B_{2} B_{1}} \cdot \frac{\sigma_{Y}}{2^{2}}=9 \sigma_{Y} \quad \text { and } \quad \frac{B_{4}-B_{2}}{B_{4} B_{2}}=0
$$

and Theorem 5.5 implies Theorem 5.7 in these two cases.

The case $k>2$ will follow from a result of Wall, a fact about Bernoulli numbers (see Theorem A.1 in the appendix) and from the evenness of $\sigma_{Y}$. As in Remark 5.6, we regard $\tau_{Y *}$ as a cohomology class $\tau_{Y *} \in H^{4 k}(Y)$ and define the integer

$$
\tau_{Y}^{2}:=\left\langle\left(\tau_{Y *}\right)^{2},[Y]\right\rangle \text {. }
$$

Setting $a_{k}:=\frac{1}{2}\left(3-(-1)^{k}\right)$, Wall $\left[31,(15)_{m}\right]$ proved that the $\widehat{A}$-genus of a $(4 k-1)-$ connected $8 k$-manifold $Y$ is given by

$$
\widehat{A}_{2 k}(Y)=\frac{a_{k}^{2} \cdot 2^{4 k-4} \cdot B_{k}^{2} \cdot\left(2^{2 k}-1\right)^{2} \cdot \tau_{Y}^{2}-k^{2} \sigma_{Y}}{2^{4 k+1} \cdot k^{2} \cdot\left(2^{4 k-1}-1\right)},
$$

where $\tau_{Y}^{2}=\chi^{2}$ in Wall's notation. Now let $B_{k}=N_{k} / D_{k}$, where $N_{k}$ and $D_{k}$ denote respectively the numerator and denominator of $B_{k}$ expressed in lowest terms. We recall from [27, page 284] that $N_{k}$ is odd and that $D_{k}=2 D_{k}^{\prime}$ where $D_{k}^{\prime}$ is odd: in fact $D_{k}^{\prime}$ is the product of odd primes $p$ such that $(p-1)$ divides $2 k$. Writing $k=2^{j} \cdot c$ for $c$ an odd integer, $j \geq 0$, we rewrite (4) as

$$
\widehat{A}_{2 k}(Y)=\frac{a_{k}^{2} \cdot 2^{4 k-6-2 j} \cdot N_{k}^{2} \cdot\left(2^{2 k}-1\right)^{2} \cdot \tau_{Y}^{2}-\left(D_{k}^{\prime}\right)^{2} \cdot c^{2} \cdot \sigma_{Y}}{2^{4 k+1} \cdot c^{2} \cdot\left(D_{k}^{\prime}\right)^{2} \cdot\left(2^{4 k-1}-1\right)} .
$$

Since $k>2$ and $Y$ is $(4 k-1)$-connected, the intersection form of $Y$ is even by [31], and hence $\tau_{Y}^{2}$ is an even integer. In addition, if $k$ is even, then for $Y$ to admit a stably complex structure, $\tau_{Y}$ must lie in $2 H^{4 k}(Y)$, and so 8 divides $\tau_{Y}^{2}$. Since $\widehat{A}_{2 k}(Y)$ is an integer, (5) entails that $2^{4 k-3-2 j}$ divides $\sigma_{Y}$.

To apply Theorem 5.5 when $k$ is odd, we must show that

$$
\operatorname{Num}\left(\frac{B_{2 k}+B_{k}}{B_{2 k} B_{k}} \cdot \frac{\sigma_{Y}}{2^{4 k-2}}\right)
$$

is even, where $\operatorname{Num}(a / b)$ denotes the numerator of $a / b$, expressed in lowest terms. Since $k$ is odd, $j=0$, and hence $2^{4 k-3}$ divides $\sigma_{Y}$. Furthermore, the largest power of 2 which divides $\operatorname{Denom}\left(\sigma_{Y} / 2^{4 k-2}\right)$ is 2 . But

$$
\operatorname{Num}\left(\frac{B_{2 k}+B_{k}}{B_{2 k} B_{k}}\right)=D_{k} N_{2 k}+D_{2 k} N_{k}=2\left(D_{k}^{\prime} N_{2 k}+D_{2 k}^{\prime} N_{k}\right)
$$

is divisible by $2^{2}$, and Theorem 5.5(1) holds. 
To apply Theorem 5.5 when $k$ is even, we must show that

$$
\operatorname{Num}\left(\frac{B_{2 k}-B_{k}}{B_{2 k} B_{k}} \cdot \frac{4 k \cdot \sigma_{Y}}{2^{4 k}}\right)
$$

is even. Since $k=2^{j} c$ and $2^{4 k-3-2 j}$ divides $\sigma_{Y}$, the largest power of 2 which can divide $\operatorname{Denom}\left(4 k \cdot \sigma_{Y} / 2^{4 k}\right)$ is $2^{j+1}$. By Theorem A.1, $2^{j+3} \operatorname{divides} \operatorname{Num}\left(B_{2 k}-\right.$ $\left.B_{k} /\left(B_{2 k} B_{k}\right)\right)$, which ensures that Theorem 5.5(2) holds.

To prove Theorem 5.1, we shall need the following result.

Lemma 5.9 Let $q \equiv 3 \bmod 4$ and let $(W, J)$ be an almost complex $(2 q+2)-$ manifold with $\partial W=S^{2 q+1}$. The stable complex structure induced on the boundary, $\left(S^{2 q+1}, S \partial J\right)$, is independent on the choice of the almost complex structure $J$ up to homotopy and depends only on the oriented diffeomorphism type of $W$.

Proof Let $\left(W, J_{0}\right)$ and $\left(W, J_{1}\right)$ be two almost complex structures on the same topological Stein filling of $S^{2 q+1}$, which is then a $q$-connected manifold. By the Hurewicz Theorem and repeated application of the Whitney trick we can find a basis of $H_{q+1}(W)$ consisting of primitive elements $\left\{x_{1}, \ldots, x_{n}\right\}$ represented by embeddings $f_{i}: S^{q+1} \hookrightarrow W$. The embedded spheres $f_{i}\left(S^{q+1}\right)$ will intersect in the pattern determined by the intersection form of $W$, which is unimodular, since the boundary of $M$ is a sphere, and is denoted by

$$
\lambda_{W}: H_{q+1}(W) \times H_{q+1}(W) \rightarrow \mathbb{Z} .
$$

We now consider the boundary connected sum $\left(W \downarrow(-W), J_{0} \downarrow\left(-J_{1}\right)\right)$, given by reversing the orientation on $\left(W, J_{1}\right)$ and then attaching an almost complex 1-handle. This manifold is again $q$-connected. By tubing together the two copies of the embeddings $f_{i}$, and taking care to reverse the orientation along the tube, we obtain embeddings $f_{i} \#\left(-f_{i}\right): S^{q+1} \hookrightarrow W \bigsqcup(-W)$, which represent a basis of the antidiagonal summand

$$
L:=\left\langle\left(x_{1},-x_{1}\right) \ldots,\left(x_{n},-x_{n}\right)\right\rangle \subset H_{q+1}(W দ(-W))=H_{q+1}(W) \oplus H_{q+1}(W) .
$$

We claim that this basis for $L$ is represented by disjoint embedded $(q+1)$-spheres with trivial normal bundle. The normal bundle of each embedding $f_{i} \#\left(-f_{i}\right), i=1, \ldots, n$, is isomorphic to the Whitney sum of the normal bundle of $f_{i}$ and its inverse, and so is trivial. Moreover, the intersection form of $W \downarrow(-W)$ is the orthogonal sum $\lambda_{W} \oplus-\lambda_{W}$, and so for all pairs $(i, j)$, the algebraic intersection of $\left(x_{i},-x_{i}\right)$ with $\left(x_{j},-x_{j}\right)$ is given by

$$
\lambda_{W \natural(-W)}\left(\left(x_{i},-x_{i}\right),\left(x_{j},-x_{j}\right)\right)=\lambda_{W}\left(x_{i}, x_{j}\right)-\lambda_{W}\left(-x_{i},-x_{j}\right)=0 .
$$


By further applications of the Whitney trick, we arrive at the required disjoint embeddings $\phi_{i}: D^{q+1} \times S^{q+1} \hookrightarrow W দ(-W)$ representing the given basis of $L$.

The stable complex structure induced on $\phi_{i}\left(D^{q+1} \times S^{q+1}\right)$ by $\zeta=S\left(J_{0} \curvearrowleft\left(-J_{1}\right)\right)$ may be regarded as an element $\zeta_{i} \in \pi_{q+1}(\mathrm{SO} / U)$ and since $q \equiv 3 \bmod 4$, each $\zeta_{i}$ lies in the image of the map $\pi_{q+1}(\mathrm{SO}) \rightarrow \pi_{q+1}(\mathrm{SO} / U)$. Moreover, the condition that $q \equiv$ 3 mod 4 implies that the stabilization homomorphism $\pi_{q+1}(\mathrm{SO}(q+1)) \rightarrow \pi_{q+1}(\mathrm{SO})$ is onto by [21], and hence we may reframe our embeddings to obtain new embeddings $\bar{\phi}_{i}$ so that each $\zeta_{i}$ is trivial. It follows that there is no obstruction to extending the stable complex structure induced by $J_{0} \downarrow\left(-J_{1}\right)$ on $W \natural(-W)$ over a handle attachment along $\bar{\phi}_{i}$. That is, we may perform stably complex surgeries on the embeddings $\bar{\phi}_{i}$ : see [6, Section 2.3]. The trace of these surgeries is a stably complex bordism, relative to the boundary, to a simply connected homology ball, which is in turn a topological ball: see [22, Lemma 7.1]. Moreover, the stable almost complex structure on the boundary is equal to the stabilization of $\partial J_{0} \#\left(-\partial J_{1}\right)$. It follows that $S\left(\partial J_{0} \#\left(-\partial J_{1}\right)\right)$ is the standard stable complex structure and thus that $S \partial J_{0}=S \partial J_{1}$.

Proof of Theorem 5.1 Suppose that $k \geq 2$. Let $(W, J)$ be a Stein filling with boundary $S^{8 k-1}$, and consider the smooth closed oriented manifold $X$ obtained by adding the $8 k$-disc to $W$ via the identity map:

$$
X:=W \cup_{\mathrm{Id}} D^{8 k} .
$$

Note that since $W$ admits an almost complex structure $J$ by hypothesis, we have $\operatorname{Im}\left(\tau_{X *}\right)=\operatorname{Im}\left(\tau_{W *}\right) \subset F_{*}\left(\pi_{4 k}(B U)\right)$. It follows from Theorem 5.7 that $X$ also admits a stable complex structure $\zeta_{X}$.

Now take the resulting stably complex manifold $\left(X, \zeta_{X}\right)$ and remove a small open disc. The outcome is a smooth oriented manifold diffeomorphic to $W$ with an induced stable complex structure $\zeta_{W}$. Since $\zeta_{W}$ extends to $X$, we conclude that the induced stable complex structure $\partial \zeta_{W}$ on $S^{8 k-1}$ is homotopic to $\zeta_{0}$. Now by Lemma 5.9, the stable complex structures $S \partial J$ and $\partial \zeta_{W}$ are homotopic and hence $S \partial J$ is homotopic to $\zeta_{0}$. This shows that only the standard stable complex structure on $S^{8 k-1}$ admits a Stein filling, which proves Theorem 5.1.

\subsection{A description of $\left(S^{8 k-1}, \varphi_{\mathrm{ex}}\right)$}

In this subsection we give an explicit description of an almost contact structure $\varphi_{\mathrm{ex}}$ on $S^{8 k-1}$ which stabilizes to $\left(S^{8 k-1}, \zeta_{\mathrm{ex}}\right)$ when $k \geq 2$. Recall that $\pi_{8 k-1}(\mathrm{SO}) \rightarrow$ $\pi_{8 k-1}(\mathrm{SO} / U)$ is onto, and that by [21], for $k \geq 2$, the stabilization homomorphism

$$
\pi_{8 k-1}(\mathrm{SO}(8 k-2)) \rightarrow \pi_{8 k-1}(\mathrm{SO})
$$


is also onto. Let $f:\left(D^{8 k-1}, S^{8 k-2}\right) \rightarrow(\mathrm{SO}(8 k-2)$, id) be a smooth map representing a class $[f] \in \pi_{8 k-1}(\mathrm{SO}(8 k-2))$, where $[f]$ stabilizes to a generator of $\pi_{8 k-1}(\mathrm{SO})$. Let $\xi_{\text {std }} \subset T S^{8 k-1}$ be the oriented hyperplane distribution given by the standard contact structure on $S^{8 k-1}$ and let $J_{\text {std }}$ be the complex structure on $\xi_{\text {std }}$ induced by the choice of a contact form. We observe that we can use $f$ to define a vector bundle automorphism

$$
\alpha_{f}: \xi_{\text {std }} \cong \xi_{\text {std }},
$$

where $\alpha_{f}$ is the identity on all fibers outside a small $(8 k-1)-$ disc $D \subset S^{8 k-1}$ and on

$$
\left.T S^{8 k-1}\right|_{D} \cong D \times \mathbb{R}^{8 k-1}
$$

we use $f$ to twist $\xi_{\text {std }}$ in the obvious way. We can then use $\alpha_{f}$ to pull-back the complex structure $J_{\text {std }}$ on $\xi_{\text {std }}$ and obtain $\alpha_{f}^{*}\left(J_{\text {std }}\right)$. Clearly $\left(\xi_{\text {std }}, J_{\text {std }}\right)$ and $\left(\xi_{\text {std }}, \alpha_{f}^{*}\left(J_{\text {std }}\right)\right)$ are isomorphic complex vector bundles but since $\alpha_{f}$ is not homotopic to a unitary automorphism of $\left(\xi_{\text {std }}, J_{\text {std }}\right)$, it follows that $\left(\xi_{\text {std }}, J_{\text {std }}\right)$ and $\left(\xi_{\text {std }}, \alpha_{f}^{*}\left(J_{\text {std }}\right)\right)$ are not homotopic as complex structures on $\xi_{\text {std }}$. Indeed, even after stabilization $\alpha_{f}$ is not homotopic to a unitary automorphism and so the almost contact structure

$$
\varphi_{\mathrm{ex}}:=\left(\xi_{\mathrm{std}} \subset T S^{8 k-1}, \alpha_{f}^{*}\left(J_{\mathrm{std}}\right)\right)
$$

stabilizes to the stable complex structure on $S^{8 k-1}$ given by acting on $\zeta_{\text {std }}$ with the generator of $\pi_{8 k-1}(\mathrm{SO} / U) \cong \mathbb{Z} / 2$. Hence we have proven the following lemma.

Lemma 5.10 For $k \geq 2$, the almost contact structure $\left(S^{8 k-1}, \varphi_{\mathrm{ex}}\right)$ of (6) stabilizes to the stable complex structure $\left(S^{8 k-1}, \zeta_{\mathrm{ex}}\right)$.

The examples $\left(S^{8 k-1}, \varphi_{\mathrm{ex}}\right)$ above and also the examples $\left(S T S^{4 k-1}, \varphi\right)$ with $c_{k}(\varphi) \neq$ 0 from Lemma 4.1 are interesting examples of $(q-1)$-connected $(2 q+1)$-dimensional almost contact manifolds which are not Stein fillable. The Stein fillability of such manifolds was studied in $[13 ; 14]$. In [5] we take up this question in the context of Theorem 2.12 by systematically studying the bordism groups $\Omega_{2 q+1}\left(B_{\zeta}^{q-1} ; \eta_{\zeta}^{q-1}\right)$.

\section{Appendix: 2-adic valuation of differences of the Bernoulli numbers}

Let $B_{k}$ be the $k^{\text {th }}$ Bernoulli number with topologist's indexing and sign conventions as in [18, page 12] and [27, Appendix B]. In particular, we have

$$
\begin{array}{llll}
B_{1}=\frac{1}{6}, & B_{2}=\frac{1}{30}, & B_{3}=\frac{1}{42}, & B_{4}=\frac{1}{30}, \\
B_{5}=\frac{5}{66}, & B_{6}=\frac{691}{2730}, & B_{7}=\frac{7}{6}, & B_{8}=\frac{3617}{510} .
\end{array}
$$


Given a fraction $a / b$, let $\operatorname{Num}(a / b)$ and $\operatorname{Denom}(a / b)$ denote respectively the numerator and the denominator of $a / b$, when expressed in lowest terms. In this appendix we prove the following theorem about Bernoulli numbers, which is the essential numbertheoretic input to the proof of Theorem 5.7.

Theorem A.1 Suppose that $k$ is even and write $k=2^{j} c$, where $c$ is odd and $j \geq 1$. Then

$$
2^{j+3} \mid \operatorname{Num}\left(\frac{B_{2 k}-B_{k}}{B_{2 k} B_{k}}\right) \text {. }
$$

Let $p$ be any prime and let $\mathbb{Z}_{p}$ denote the ring of $p$-adic integers. As usual, define the $p$-adic valuation of $s \in \mathbb{Z}_{p}$ by $\operatorname{ord}_{p} s$, such that $s=u p^{\operatorname{ord}_{p} s}$ where $u \in \mathbb{Z}_{p}^{\times}$is a unit.

We will prove Theorem A.1 later, since we first need to show some $p$-adic properties of the Bernoulli numbers. From now on, it is more convenient to switch to the notation of signed and even-indexed Bernoulli numbers $\mathcal{B}_{n}$ as commonly used in number theory. They may be defined by the generating function

$$
\frac{t}{e^{t}-1}=\sum_{n \geq 0} \mathcal{B}_{n} \frac{t^{n}}{n !}, \quad|t|<2 \pi .
$$

These numbers are rational and $\mathcal{B}_{n}=0$ for odd $n>1$. The even-indexed Bernoulli numbers alternate in sign, such that $(-1)^{n / 2+1} \mathcal{B}_{n}>0$ for even $n>0$. Accordingly

$$
(-1)^{n+1} B_{n}=\mathcal{B}_{2 n}, \quad n \geq 1 .
$$

The famous theorem of von Staudt and Clausen [20, Theorem 3, page 233] asserts, for even $n \geq 2$, that

$$
\mathcal{B}_{n}+\sum_{p-1 \mid n} \frac{1}{p} \in \mathbb{Z}, \quad \text { which implies that } \operatorname{Denom}\left(\mathcal{B}_{n}\right)=\prod_{p-1 \mid n} p .
$$

Let $n \geq 2$ be even. If $p-1 \mid n$, then we obtain by (7) that

$$
\mathcal{B}_{n}+\frac{1}{p} \in \mathbb{Z}_{p}
$$

whereas we already have $\mathcal{B}_{n} \in \mathbb{Z}_{p}$ in the case $p-1 \nmid n$. Both cases imply that

$$
\mathcal{B}_{n}-\mathcal{B}_{m} \in \mathbb{Z}_{p},
$$

whenever $n, m \geq 2$ are both even and satisfy $n \equiv m \bmod p-1$. As an easy consequence, iterated finite differences of a sequence of Bernoulli numbers $\mathcal{B}_{n}$ are $p$-integers, assuming that all indices are even and congruent $\bmod p-1$. Now, we consider the special case $p=2$, where we use the following more general result of Carlitz. 
Theorem A.2 (Carlitz [8, Theorem 7]) If $n \geq 2$ is even, $r \geq 1$, and $2^{e-1} \mid w$ with $e \geq 2$, then

$$
\sum_{s=0}^{r}(-1)^{s}\left(\begin{array}{l}
r \\
s
\end{array}\right) 2 \mathcal{B}_{n+s w} \equiv 0 \bmod \operatorname{gcd}\left(2^{n-1}, 2^{r e+\lambda}\right),
$$

where $\lambda=\min \left(r-1, r-r^{\prime}+3\right)$ and $2^{r^{\prime}} \leq 2 r<2^{r^{\prime}+1}$.

Note that the sum above describes an iterated finite difference with increment $w$. As mentioned above, this sum still lies in $\mathbb{Z}_{2}$, if we cancel the factor 2 that occurs. We can rewrite this result as follows.

Corollary A.3 If $n, w \geq 2$ are both even and $r \geq 1$, then

$$
\operatorname{ord}_{2}\left(\sum_{s=0}^{r}(-1)^{s}\left(\begin{array}{l}
r \\
s
\end{array}\right) \mathcal{B}_{n+s w}\right) \geq \min (n-2, r e+\lambda-1),
$$

where $e=1+\operatorname{ord}_{2} w \geq 2, l=\left\lfloor\log _{2} r\right\rfloor \geq 0$, and $\lambda=\min (r-1, r-l+2) \geq 0$.

Proposition A.4 If $m>n \geq 2$ are both even, then

$$
\operatorname{ord}_{2}\left(\frac{1}{\mathcal{B}_{n}}-\frac{1}{\mathcal{B}_{m}}\right)=2+\operatorname{ord}_{2}\left(\mathcal{B}_{n}-\mathcal{B}_{m}\right) \geq \min \left(n, 2+\operatorname{ord}_{2}(m-n)\right) .
$$

Proof We first observe that

$$
\operatorname{ord}_{2}\left(\frac{1}{\mathcal{B}_{n}}-\frac{1}{\mathcal{B}_{m}}\right)=\operatorname{ord}_{2}\left(\frac{\mathcal{B}_{n}-\mathcal{B}_{m}}{\mathcal{B}_{n} \mathcal{B}_{m}}\right)=2+\operatorname{ord}_{2}\left(\mathcal{B}_{n}-\mathcal{B}_{m}\right),
$$

since by $(7)$ we have $\operatorname{ord}_{2}\left(\operatorname{Denom}\left(\mathcal{B}_{n} \mathcal{B}_{m}\right)\right)=2$. Using Corollary A.3 with parameters $r=1$ and $w=m-n$, we then infer that

$$
\operatorname{ord}_{2}\left(\mathcal{B}_{n}-\mathcal{B}_{m}\right) \geq \min \left(n-2, \operatorname{ord}_{2}(m-n)\right),
$$

completing the proof.

Proof of Theorem A.1 Recall that $k=2^{j} c$, where $c$ is odd and $j \geq 1$. Since $k$ is even, the Bernoulli numbers $\mathcal{B}_{2 k}$ and $\mathcal{B}_{4 k}$ have the same sign. Thus, we can apply Proposition A.4 to obtain that

$$
\operatorname{ord}_{2}\left(\frac{B_{2 k}-B_{k}}{B_{2 k} B_{k}}\right)=2+\operatorname{ord}_{2}\left(\mathcal{B}_{2 k}-\mathcal{B}_{4 k}\right) \geq \min \left(2 k, 3+\operatorname{ord}_{2} k\right)=3+\operatorname{ord}_{2} k .
$$

The last step follows by a simple counting argument. Since $\operatorname{ord}_{2} k=j$, this gives the result. 
In the summer of 2013, Theorem A.1 arose as a conjecture. At the same time, it was independently proved by Karl Dilcher and the author of this appendix using results of Carlitz.

\section{References}

[1] H J Baues, Obstruction theory on homotopy classification of maps, Lecture Notes in Mathematics 628, Springer, Berlin (1977) MR0467748

[2] MS Borman, Y Eliashberg, E Murphy, Existence and classification of overtwisted contact structures in all dimensions arXiv: 1404.6157

[3] R Bott, The stable homotopy of the classical groups, Ann. of Math. 70 (1959) 313-337 MR0110104

[4] F Bourgeois, Odd-dimensional tori are contact manifolds, Int. Math. Res. Not. 2002 (2002) 1571-1574 MR1912277

[5] J Bowden, D Crowley, A I Stipsicz, The topology of Stein fillable manifolds in high dimensions, III in preparation

[6] J Bowden, D Crowley, A I Stipsicz, Contact structures on $M \times S^{2}$, Math. Ann. 358 (2014) 351-359 MR3158000

[7] J Bowden, D Crowley, A I Stipsicz, The topology of Stein fillable manifolds in high dimensions, I, Proc. Lond. Math. Soc. 109 (2014) 1363-1401 MR3293153

[8] L Carlitz, Kummer's congruence for the Bernoulli numbers, Portugal. Math. 19 (1960) 203-210 MR0125054

[9] K Cieliebak, Y Eliashberg, From Stein to Weinstein and back: Symplectic geometry of affine complex manifolds, Amer. Math. Soc. Colloq. Publ. 59, Amer. Math. Soc. (2012)

[10] Y Eliashberg, Filling by holomorphic discs and its applications, from: "Geometry of low-dimensional manifolds, 2", (S K Donaldson, C B Thomas, editors), London Math. Soc. Lecture Note Ser. 151, Cambridge Univ. Press (1990) 45-67 MR1171908

[11] Y Eliashberg, Topological characterization of Stein manifolds of dimension $>2$, Internat. J. Math. 1 (1990) 29-46 MR1044658

[12] J B Etnyre, K Honda, On symplectic cobordisms, Math. Ann. 323 (2002) 31-39 MR1906906

[13] H Geiges, Contact structures on $(n-1)$-connected $(2 n+1)$-manifolds, Pacific J. Math. 161 (1993) 129-137 MR1237141

[14] H Geiges, Applications of contact surgery, Topol. 36 (1997) 1193-1220 MR1452848

[15] H Geiges, An introduction to contact topology, Cambridge Studies in Advanced Mathematics 109, Cambridge Univ. Press (2008) MR2397738 
[16] H Geiges, K Zehmisch, How to recognize a 4-ball when you see one, Münster J. Math. 6 (2013) 525-554 MR3148221

[17] P Ghiggini, K Niederkrüger, C Wendl, Subcritical contact surgeries and the topology of symplectic fillings arXiv:1408.1051

[18] F Hirzebruch, Topological methods in algebraic geometry, Grundl. Math. Wissen. 131, Springer, New York (1966) MR0202713

[19] D Husemoller, Fibre bundles, 3rd edition, Graduate Texts in Mathematics 20, Springer, New York (1994) MR1249482

[20] K Ireland, M Rosen, A classical introduction to modern number theory, 2nd edition, Graduate Texts in Mathematics 84, Springer, New York (1990) MR1070716

[21] M A Kervaire, Some nonstable homotopy groups of Lie groups, Illinois J. Math. 4 (1960) 161-169 MR0113237

[22] M A Kervaire, J W Milnor, Groups of homotopy spheres, I, Ann. of Math. 77 (1963) 504-537 MR0148075

[23] W S Massey, Obstructions to the existence of almost complex structures, Bull. Amer. Math. Soc. 67 (1961) 559-564 MR0133137

[24] P Massot, K Niederkrüger, C Wendl, Weak and strong fillability of higher dimensional contact manifolds, Invent. Math. 192 (2013) 287-373 MR3044125

[25] J Milnor, Lectures on the h-cobordism theorem, Princeton Univ. Press (1965) MR0190942

[26] J Milnor, E Spanier, Two remarks on fiber homotopy type, Pacific J. Math. 10 (1960) 585-590 MR0117750

[27] J W Milnor, J D Stasheff, Characteristic classes, Annals of Mathematics Studies 76, Princeton Univ. Press (1974) MR0440554

[28] S Smale, Generalized Poincaré's conjecture in dimensions greater than four, Ann. of Math. 74 (1961) 391-406 MR0137124

[29] S Smale, On the structure of 5-manifolds, Ann. of Math. 75 (1962) 38-46 MR0141133

[30] R E Stong, Notes on cobordism theory, Princeton Univ. Press (1968) MR0248858

[31] C T C Wall, Classification of $(n-1)$-connected $2 n$-manifolds, Ann. of Math. 75 (1962) 163-189 MR0145540

[32] A Weinstein, Contact surgery and symplectic handlebodies, Hokkaido Math. J. 20 (1991) 241-251 MR1114405

[33] H Whitney, The self-intersections of a smooth n-manifold in 2n-space, Ann. of Math. 45 (1944) 220-246 MR0010274

[34] H Yang, Almost complex structures on $(n-1)$-connected $2 n$-manifolds, Topology Appl. 159 (2012) 1361-1368 MR2879365 
Ludwig-Maximillians Universität, Mathemathisches Institut

Theresienstr. 39, D-80333 Munich, Germany

Institute of Mathematics, University of Aberdeen

Aberdeen AB24 3UE, UK

Rényi Institute of Mathematics, Hungarian Academy of Sciences, Réaltanoda utca 13-15, Budapest, H-1053, Hungary

Universität Göttingen, Mathematisches Institut

D-37073 Göttingen, Germany

jonathan.bowden@math.lmu.de, dcrowley@abdn.ac.uk, stipsicz.andras@renyi.mta.hu, stipsicz@renyi.hu, bk@bernoulli.org

Proposed: Yasha Eliashberg

Seconded: Peter Teichner, Simon Donaldson
Received: 28 October 2014 Revised: 23 February 2015 